MPI-Ph/92-118

December 1992

\title{
BOTTOM-UP APPROACH TO UNIFIED SUPERGRAVITY MODELS
}

\author{
Marek Olechowski \\ Insitute of Theoretical Physics \\ Warsaw University \\ ul. Hoża 69, 00-681 Warsaw, Poland \\ Stefan Pokorski 2 \\ Max-Planck-Institut für Physik \\ - Werner-Heisenberg-Institut - \\ P.O.Box 40 12 12, Münich, Fed. Rep. Germany
}

\begin{abstract}
A new approach is proposed to phenomenological study of a generic unified supergravity model, which reduces to the minimal supersymmetric standard model. The model is effectively parametrized in terms of five low energy observables. In consequence, it is easy to investigate systematically the parameter space of the model, allowed by the requirement of radiative electroweak symmetry breaking and by the present experimental limits.

Radiative corrections due to large Yukawa couplings and particle-sparticle mass splitting are included into the analysis and found to have important effects, in particular on the degree of fine tuning in the model.

In this framework there are presented the predictions of the model for various low energy physical observables and their dependence on the values of the top quark mass and $\tan \beta$ is discussed. Results are also given for the large $\tan \beta$ scenario, $\tan \beta \approx m_{t} / m_{b}$.

Our approach can be easily extended to non-minimal supergravity models, which do not assume the universality of the soft breaking parameters at the unification scale $M_{X}$. Such an extension will be particularly useful once the masses of some sparticles are known, allowing for a model independent study of the parameter space at $M_{X}$.
\end{abstract}

\footnotetext{
${ }^{1}$ Supported in part by the Polish Committee for Scientific Research

${ }^{2}$ On leave of absence from the Institute of Theoretical Physics, Warsaw University
} 


\section{Introduction}

The "supergravity inspired" minimal supersymmetric standard model (MSSM) is an interesting laboratory for studying various phenomenological aspects of supersymmetry. In this model the soft supersymmetry breaking parameters of the low energy lagrangian are given, via the renormalization group (RG) evolution, in terms of the unification scale parameters. These are: the universal gaugino and scalar masses $M_{o}$ and $m_{o}$, respectively, and the trilinear scalar coupling $A_{o}$. Apart from the gauge and Yukawa couplings there are in the model two more free parameters: the Higgs superfield mixing mass $\mu_{o}$ in the superpotential and the corresponding soft breaking parameter $B_{o}$. The actual mechanism of the soft supersymmetry breaking (and the origin of the $\mu$-term in the superpotential) are not yet understood theoretically. Although there are various theoretical suggestions with regard to the values of those parameters at the unification scale, they should be considered at present as free parameters of the model.

One of the most attractive features of the supergravity models is the possibility of radiatively induced gauge symmetry breaking ${ }^{[1]}$, which becomes manifest at the tree level after the RG evolution of the parameters of the lagrangian from the unification scale $M_{X}$ to the scale $M_{Z}$. This way the hierarchy $M_{Z} / M_{X}$ becomes related to the presence in nature of large Yukawa couplings. The minimal model supplemented by the requirement of radiative gauge symmetry breaking is a very predictive framework: with only 5 free mass parameters $\mu_{o}, m_{o}, M_{o}, A_{o}$ and $B_{o}$ (and the yet unknown top quark Yukawa coupling $\left.Y_{t}^{o}\right)$, it interconnects 1) the $S U(2) \times U(1)$ gauge symmetry breaking with $v=174 \mathrm{GeV}, 2)$ the very constrained Higgs sector of the MSSM, 3) the sparticle mass spectrum and 4) the relic density of the lightest supersymmetric particle (LSP). In addition, it has recently been strongly reemphasized that the predictivity of the model is greatly enhanced by the requirement of no fine tuning of the parameters ${ }^{[2]}$. Indeed, the requirement that the $M_{W, Z}$ be within the measured value without fine tuning of the unification scale parameters strongly limits the magnitude of the supersymmetry breaking scale and, in consequence, of the sparticle masses.

In spite of the large number of papers devoted to the model, its analysis is not yet fully satisfactory (for a complete list of earlier references we refer the reader to the ref. [3]). Its full parameter space has not been systematically explored yet. Secondly, it has recently been understood that radiative corrections originating from the combined effect of large Yukawa couplings and particle-sparticle mass splitting have important effects on the mechanism of radiatively induced $S U(2) \times$ $U(1)$ symmetry breaking ${ }^{[4]}$ and on the Higgs sector ${ }^{[5]}$. In consequence, one may expect important modifications in the predictions of the model.

There are two main points in our strategy in this paper. Firstly, we set up the formalism to explore the full parameter space of the model. Our formalism is based on the bottom to top approach whose essence is as follows: we choose a set of values for 5 low energy physical parameters, and then look for the set of parameters at the unification scale $M_{X}$ which induces radiative gauge symmetry breaking and gives the chosen low energy values. Thus, we reverse the standard procedure which is to find the low energy parameters corresponding to a given set of the boundary conditions 
at the unification scale. This way we can systematically study the phenomenology of the "supergravity inspired" MSSM compatible with the idea of radiatively induced symmetry breaking, with no additional assumptions (apart from their universality) about the values (and their correlation) of the parameters at $M_{X}$ (for which we have little theoretical insight). In this approach it is straightforward to implement all the experimental constraints on the low energy parameters and to discuss systematically the correlations between physical observables, which are predicted by the model. Effectively, the model is now parametrized by five low energy physical parameters. We would also like to stress that our approach is readily extendable to non-minimal supergravity models, which do not assume the universality of the soft supersymmetry breaking terms at the scale $M_{X}$, provided more than five low energy physical parameters is used as input. Once some sparticles are discovered and their masses known, such an extension will allow for a model independent study of the parameter space at the unification scale.

Secondly, we consistently include radiative corrections generated by large Yukawa couplings and particle-sparticle mass splitting. To this end, we use the RG equations for all parameters of the potential with heavy sparticles decoupled at their thresholds. In the context of radiative breaking of the $S U(2) \times U(1)$ symmetry this technique has already been advocated in ref.[6]. It has also been used to study similar corrections to the Higgs sector in the MSSM and found to be quite accurate ${ }^{[7]}$. In this paper we use the RG equations of ref.[6] with squarks and gluinos decoupled at $M_{S U S Y}$ (the precise definition of this scale will be discussed in Section 2). Radiative corrections generated by the other sparticle-particle mass splitting are small and can be neglected.

The question about the role of the large radiative corrections in the phenomenological analysis of the model has already been given some attention in the literature (see ref. [3] and references therein). However, to our knowledge, no fully consistent treatment of such corrections at the 1-loop level, simultaneously in all four sectors mentioned above, exists yet. Their full impact on the phenomenology of the model has become easy to explore only within our bottom to top approach.

The paper is organized as follows: In the next Section we present the details of our approach. Section 3 is devoted to a qualitative discussion of those predictions of the model which follow from the requirement of radiative gauge symmetry breaking. In Section 4 we discuss the problem of fine tuning of the parameters and emphasize the role of radiative corrections in sizably diminishing the degree of fine tuning. In Section 5 we present our results, after imposing the presently available experimental constraints. Finally, Section 6 contains a very brief discussion of the large $\tan \beta$ scenario and a summary of this work is given in Section 7.

\section{The Bottom-Up Approach}

The details of our procedure are as follows. We begin with choosing, in addition to the "experimentally" known quantities

$$
\begin{aligned}
& \alpha_{E M}\left(M_{Z}\right)=1 / 127.9, \quad \sin ^{2} \theta_{W}\left(M_{Z}\right)=0.2324 \\
& m_{\tau}\left(M_{Z}\right)=1.746 \mathrm{GeV}, \quad M_{Z}=91.19 \mathrm{GeV},
\end{aligned}
$$


a set of values for the following low energy unknown parameters:

$$
M_{t}, \quad M_{A}, \quad \tan \beta, \quad m_{Q}^{2}, \quad m_{U}^{2}
$$

where $M_{A}$ is the Higgs pseudoscalar mass and $m_{Q}^{2}$ and $m_{U}^{2}$ are the soft (low energy) mass parameters of the squarks of the third generation. The physical squark masses are eigenvalues of the mass matrix. For the bottom to top approach this is the most economical choice of the low energy input parameters: $M_{Z}, \tan \beta$ and $M_{A}$ are directly related to the parameters of the tree level potential and the squark mass parameters determine the magnitude of the dominant radiative corrections to the scalar quartic couplings. So they must be fixed anyway at the begining of the analysis. To proceed further we have to discuss in some detail the unification of gauge and Yukawa couplings.

\subsection{Unification of gauge and Yukawa couplings}

The analysis of the unification of couplings in the MSSM have been addressed in a number of papers ${ }^{[8]}$, with the most recent and thorough discussion given in ref. $[9,10]$. At the 2-loop level, the RG equations for the gauge and Yukawa couplings are a coupled set of equations. However, in the calculation of the gauge couplings, it is a very good approximation to set the top quark Yukawa coupling to be constant ${ }^{[9]}$ (for $\tan \beta \approx m_{t} / m_{b}$, the b-quark Yukawa coupling is as large as the t-quark coupling and the same procedure must be applied to both of them). Then the effect of the Yukawa couplings on the running of $\alpha_{i}$ is realized as a negative correction to the 1-loop coefficients $b_{i}$ 's (which is, anyway, small; see below). Effectively, for some chosen value of $Y_{t}$, we can first discuss the gauge coupling unification. Starting from the experimental values for $\alpha_{E M}\left(M_{Z}\right)$ and $\sin ^{2} \theta_{W}\left(M_{Z}\right)$, we use the unification conditions to predict the values of the running strong coupling constant $\alpha_{3}(Q)$ and the unification scale $M_{X}$ (for the purpose of this paper we neglect the experimental uncertainity in the determination of $\sin ^{2} \theta_{W}$ ). After the inclusion of the 1-loop threshold corrections coming from the supersymmetric particles, we obtain for each gauge coupling the relation

$$
\frac{1}{\alpha_{i}(Q)}=\frac{1}{\alpha_{i}\left(M_{X}\right)}+\frac{b_{i}{ }^{M S S M}}{2 \pi} \ln \left(\frac{M_{X}}{Q}\right)+\gamma_{i}+\frac{1}{\alpha_{i}^{t h}}+\Delta_{i}(Q)
$$

where $\gamma_{i}$ contains the 2 -loop contributions to the beta functions, and

$$
\frac{1}{\alpha_{i}^{t h}}=\sum_{\eta, M_{\eta}>Q} \frac{b_{i}^{\eta}}{2 \pi} \ln \left(\frac{M_{\eta}}{Q}\right)
$$

is the 1 -loop threshold correction to $1 / \alpha_{i}(Q)$. The summation is over all sparticle and heavy Higgs doublet states with masses above $Q ; b_{i}^{\eta}$ is the contribution of each of those particles to the 1-loop beta function coefficient of the gauge coupling $\alpha_{i}$ (when solving the RG equations at the 2-loop level, it is sufficient to consider threshold corrections at the 1-loop level). The $\Delta_{i}$ includes the threshold correction related to the top quark mass and the effect of the $Y_{t}$ on the running of $\alpha_{i}$. The importance of various terms in eq. (2.3) can be illustrated by considering their contribution to 
the value of $\alpha_{3}\left(M_{Z}\right)$, obtained from the unification conditions. It is well known that the 2-loop corrections $\gamma_{i}$ give $\sim 10 \%$ increase in the value of $\alpha_{3}\left(M_{Z}\right)$ as compared to the 1-loop result and must be included into the analysis. The 2-loop top quark Yukawa coupling corrections to $\alpha_{3}\left(M_{Z}\right)$, included in $\Delta_{i}$ 's, give $-0.001,-0.0015$, $0.002,-0.003$ for $Y_{t}^{2}\left(M_{X}\right) / 4 \pi \simeq 0.1,0.2,0.4,1.0$, respectively ${ }^{[10]}$ and the top quark threshold correction is even smaller, (and of opposite sign) or at most similar, for $M_{t}<200 \mathrm{GeV}^{[9]}$. Altogether, for the range of the top quark masses and Yukawa couplings considered in this paper, those corrections do not exceed (1-2)\% and we neglect them in our analysis.

The contribution of the sparticle thresholds to $1 / \alpha_{3}\left(M_{Z}\right)$ can be effectively parametrized in terms of only one parameter $T_{S U S Y}$ as follows:

$$
\Delta^{t h}\left(\frac{1}{\alpha_{3}\left(M_{Z}\right)}\right)=\frac{1}{2 \pi} \ln \frac{T_{S U S Y}}{M_{Z}}
$$

where the scale $T_{S U S Y}$ has the obvious interpretation in the (unrealistic) case of the fully degenerate sparticle spectrum: it is just the common mass of all the sparticles. Notice that, in this case, a non-zero threshold corrections to $1 / \alpha_{i}\left(M_{Z}\right)$ are present only if $T_{S U S Y}>M_{Z}$ and, in consequence, the contribution (2.5) implies a decrease in the value of $\alpha_{3}\left(M_{Z}\right)$.

In the realistic case, in the presence of sparticle mass splitting, and in the approximation in which one neglects the effects of the electroweak symmetry breaking on the sparticle masses, i.e. assuming no mixing, the scale $T_{S U S Y} \operatorname{reads}^{[10]}$ :

$$
T_{S U S Y}=m_{\tilde{H}}\left(\frac{m_{\tilde{W}}}{m_{\tilde{g}}}\right)^{28 / 19}\left(\frac{m_{\tilde{l}}}{m_{\tilde{q}}}\right)^{3 / 19}\left(\frac{m_{H}}{m_{\tilde{H}}}\right)^{3 / 19}\left(\frac{m_{\tilde{W}}}{m_{\tilde{H}}}\right)^{4 / 19}
$$

where $m_{\tilde{q}}, m_{\tilde{g}}, m_{\tilde{l}}, m_{\tilde{W}}, m_{\tilde{H}}$ and $m_{H}$ are the characteristic masses of the squarks, gluinos, sleptons, electroweak gauginos, Higgsinos and the heavy Higgs doublet, respectively. Here we have assumed that all the sparticle masses are larger than $M_{Z}$ (in the approximation of no mixing); if any sparticle mass is smaller then $M_{Z}$, it should be replaced by $M_{Z}$ when applying eq. (2.6). It is obvious that $T_{S U S Y}$ depends strongly only on the first two factors and in the model under investigation, with gaugino masses coming from a common supersymmetry breaking scale $M_{o}$ at $M_{X}, T_{S U S Y}$ is well approximated by ${ }^{[10]}$ :

$$
T_{S U S Y} \approx m_{\tilde{H}}\left(\frac{\alpha_{2}\left(M_{Z}\right)}{\alpha_{3}\left(M_{Z}\right)}\right)^{3 / 2} \approx \mu\left(\frac{\alpha_{2}\left(M_{Z}\right)}{\alpha_{3}\left(M_{Z}\right)}\right)^{3 / 2}
$$

Clearly, unless the Higgs mixing parameter in the superpotential $\mu>600 \mathrm{GeV}$, the effective scale $T_{S U S Y}<M_{Z}$ and the sparticle threshold corrections tend to increase the value of $\alpha_{3}$. As it will be discussed later on, large values of $\mu$ are less natural and the most interesting range is $100 \mathrm{GeV}<\mu<600 \mathrm{GeV}$. Therefore, for our model, the typical values are $15 \mathrm{GeV}<T_{S U S Y}<M_{Z}$. The corresponding values of $\alpha_{3}\left(M_{Z}\right)$ obtaind from the unification condition vary from $\alpha_{3}\left(M_{Z}\right)=0.123$ for $T_{S U S Y}=M_{Z}$ (i.e. effectively, threshold corrections to $\alpha_{3}\left(M_{Z}\right)$ cancell out) to $\alpha_{3}\left(M_{Z}\right)=0.127$ for $T_{S U S Y}=15 \mathrm{GeV}$ (this case can be realized in practice e.g. by decoupling the 
colour sparticles in the RG equations for $\alpha_{i}$ 's at $m_{c o l} \approx 3 M_{Z}$ and the remaining sparticles at $M_{Z}$ (see eq, (2.6))). Experimentally, $\alpha_{3}\left(M_{Z}\right)$ is poorly determined and, conservatively, any value in the range $(0.12 \pm 0.01)$ is still acceptable. We see from the above discussion that the sparticle spectra predicted by the model push us into the upper range of values for $\alpha_{3}\left(M_{Z}\right)$, unless we take less natural solutions. Of course, for full consistency, one should take care of the fact that the threshold corrections correlate the value of $\alpha_{3}$ with the specific solution obtained for the parameters of the model ( $\mu$ in particular) which, in turn, slightly depend on the value of $\alpha_{3}\left(M_{Z}\right)$. However, there is an inherent uncertainity in the theoretical prediction for $\alpha_{3}\left(M_{Z}\right)$ which follows from the unknown contributions from the thresholds at the unification scale and from the higher dimension operators which are likely to be as important as the dependence on $T_{S U S Y}{ }^{[9]}$. Therefore, in this paper we take $T_{S U S Y}=M_{Z}$ and use for the gauge couplings the supersymmetric RG equations down to the scale $M_{Z}$. This gives $\alpha_{3}\left(M_{Z}\right)=0.123$, close to the central experimental value. For the unification scale we get $M_{X} \cong 2.4 \times 10^{16} \mathrm{GeV}$.

Once the running of the gauge couplings is determined, we can proceed with the determination of the top, bottom and $\tau$ Yukawa couplings in the whole range $\left(M_{Z}, M_{X}\right)$. In our procedure the $Y_{\tau}$ and $Y_{t}$ at $M_{Z}$ are fixed by the values of the physical $\tau$ mass $M_{\tau}$ and the chosen physical top quark mass $M_{t}$ and the value of $\tan \beta$. The running mass $m_{t}\left(M_{t}\right)$ is related to the physical mass (defined as the pole of the propagator) by the following 1 -loop formula ${ }^{[11]}$ :

$$
m_{q}\left(M_{q}\right)=\frac{M_{q}}{1+\frac{4 \alpha_{3}\left(M_{q}\right)}{3 \pi}}
$$

For the running of $m_{t}\left(M_{t}\right)$ down to $M_{Z}$ we use the 2-loop RG equations of the MSSM and we obtain $Y_{t}\left(M_{Z}\right)$ from the equation $m_{t}\left(M_{Z}\right)=Y_{t}\left(M_{Z}\right) v / \sqrt{1+\cot ^{2} \beta}$. The running of the couplings $Y_{t}, Y_{b}, Y_{\tau}$ in the range $\left(M_{Z}, M_{X}\right)$ is obtained by solving the 2-loop coupled RG equations with the boundary condition $Y_{b}^{o}=Y_{\tau}^{o}$ at $M_{X}$. The solutions to the RG equations with those mixed (low and high energy) boundary conditions are obtained by iteration. For consistency with the RG equations for the gauge couplings we use the supersymmetric RG equations for the Yukawa couplings in the whole range $\left(M_{Z}, M_{X}\right)$. Indeed, it has recently been shown ${ }^{[10]}$ that the same effective scale, $T_{S U S Y}$, governs the supersymmetric particle threshold corrections to the gauge and Yukawa couplings.

The reason for taking the top and not the bottom mass as the input parameter is as follows: as soon as the top quark is discovered, its mass will be known with good precision whereas the physical bottom mass is not directly accessible to experiment and its value will remain very uncertain and model dependent. In this paper we present the results for the following sets of $\left(M_{t}, \tan \beta\right)$ values: a) $\left.130,2, \mathrm{~b}\right) 130$, 10 , c) 180,2 , d) 180,30 , e) 130,31 and f) 180,51 (all masses given in GeV). It is interesting to comment on the predictions for the bottom quark mass obtained in each of those cases. They are summarized below: 


\begin{tabular}{c|ccc}
\hline & $m_{b}\left(M_{Z}\right)$ & $m_{b}\left(M_{b}\right)$ & $M_{b}$ \\
\hline $\mathrm{a}$ & 4.01 & 5.57 & 6.08 \\
$\mathrm{~b}$ & 4.04 & 5.60 & 6.12 \\
$\mathrm{c}$ & 3.30 & 4.71 & 5.16 \\
$\mathrm{~d}$ & 3.55 & 5.02 & 5.49 \\
$\mathrm{e}$ & 3.81 & 5.33 & 5.82 \\
$\mathrm{f}$ & 3.17 & 4.56 & 5.01 \\
\hline
\end{tabular}

The running mass $m_{b}\left(M_{b}\right)$ is obtained from $m_{b}\left(M_{Z}\right)$ by means of the $S U(3) \times U(1)$ 2-loop RG equations and the physical mass $M_{b}$ is given by the eq. (2.8).

As said above, the physical bottom mass is a poorly known quantity but, still, following the particle data book it is expected to be in the range, say

$$
M_{b}=4.6-5.3 \mathrm{GeV}
$$

We see that the conditions of the gauge coupling unification and of the unification of the bottom and $\tau$ Yukawa couplings result in too large a value of the $M_{b}$, for moderately heavy top quark. It is so because the large value of $\alpha_{3}\left(M_{Z}\right)(\sim 0.12)$ gives too much of a strong correction to the running of $m_{b}\left(M_{X}\right)=m_{\tau}\left(M_{X}\right)$, which has to be partly cancelled out by large, negative contribution from the top quark Yukawa coupling. In consequence, as discussed in detail in ref. [10], in this supersymmetric grand unification scenario, the value of $M_{b}$ in the range (2.9) implies the top quark mass remarkably close to its quasi infrared fixed point value associated with the "traviality" bounds on the top Yukawa coupling. A top quark mass $M_{t} \approx 130-140 \mathrm{GeV}$ happens to be close to its infrared fixed point (i.e. consistent with (2.9)) only for values of $\tan \beta$ close to one, or very large values of $\tan \beta$, $\tan \beta \geq m_{t}\left(m_{t}\right) / m_{b}\left(m_{t}\right)$, above the value for which the unification of the three Yukawa couplings, $Y_{t}^{o}=Y_{b}^{o}=Y_{\tau}^{o}$, occurs. Those values of $\tan \beta$ are the lower and upper (or even exeeding it) limits, respectively, for which radiative gauge symmetry breaking can be realized in the model and, at best, at the expense of a rapidly increasing fine-tuning of the parameters (to be discussed later on). The situation is different for $M_{t} \sim(180-190) \mathrm{GeV}$, which corresponds to its quasi infrared fixed point value for a large range of $\tan \beta$ values.

In summary, exact unification of gauge and $\tau$ and the bottom Yukawa couplings in the MSSM, together with the range (2.9) for the bottom mass, imply that $M_{t}<$ $180 \mathrm{GeV}$ can be realized only for $\tan \beta \approx 1$ or $\tan \beta \approx m_{t}\left(m_{t}\right) / m_{b}\left(m_{t}\right)$, i.e. for values for which the mechanism of radiative gauge symmetry breaking can be realized only at the expense of a large amount of fine-tuning (or, in some cases not realized at all). From this point of view, the values $M_{t}>180 \mathrm{GeV}$ look more natural in the model. However, one should remember that the exact unification of couplings may be subject to the high energy threshold and higher dimension operator corrections, which can modify the above strong conclusions. This is true for both the gauge coupling and the $\tau$ and bottom Yukawa coupling unification condition. For instance, if $\alpha_{3}\left(M_{Z}\right)$ is smaller by $\Delta=-0.006$ (the maximum magnitude of the high energy corrections, estimated in ref. [9]) then we get the following results: 


\begin{tabular}{c|ccc}
\hline & $m_{b}\left(M_{Z}\right)$ & $m_{b}\left(M_{b}\right)$ & $M_{b}$ \\
\hline $\mathrm{a}$ & 3.88 & 5.30 & 5.75 \\
$\mathrm{~b}$ & 3.91 & 5.34 & 5.80 \\
$\mathrm{c}$ & 2.97 & 4.21 & 4.60 \\
$\mathrm{~d}$ & 3.42 & 4.76 & 5.18 \\
$\mathrm{e}$ & 3.70 & 5.09 & 5.53 \\
$\mathrm{f}$ & 3.08 & 4.35 & 4.74 \\
\hline
\end{tabular}

On the other hand, with $\alpha_{3}\left(M_{Z}\right)=0.123$ and relaxing the bottom and $\tau$ Yukawa coupling unification, for $M_{b}=5.2 \mathrm{GeV}$ one gets the following results for the ratio $r=h_{b}\left(M_{X}\right) / h_{\tau}\left(M_{X}\right)$ :

\begin{tabular}{c|c}
\hline & $\mathrm{r}$ \\
\hline $\mathrm{a}$ & 0.829 \\
$\mathrm{~b}$ & 0.820 \\
$\mathrm{c}$ & 1.008 \\
$\mathrm{~d}$ & 0.923 \\
$\mathrm{e}$ & 0.846 \\
$\mathrm{f}$ & 1.135 \\
\hline
\end{tabular}

The above examples illustrate the order of magnitude of the corrections to the exact unification conditions, which are necessary to make moderate values of the top quark mass and $\tan \beta$ compatible with the range of values (2.9) for the physical bottom mass.

In view of all the uncertainities discussed above, in this paper we present the results for all cases $(\mathrm{a}-\mathrm{f})$, with the gauge and Yukawa couplings fixed by the exact unification condition. Even in the presence in some of those cases of the likely inconsistency with the experimental value in the predicted bottom quark mass, this is a useful exercise since it allows to discuss interesting correlations present in the model, as a function of $M_{t}$ and $\tan \beta$. Moreover, modifications to $\alpha_{3}\left(M_{Z}\right)$ and/or to the relation $Y_{\tau}\left(M_{X}\right)=Y_{b}\left(M_{X}\right)$, which are sufficient to bring down the value of $M_{b}$ into the range (2.9), have only little impact on the rest of our results.

\subsection{Determination of the parameters in the Higgs potential}

After those preliminaries we are now able to fix all the parameters in the Higgs potential at the scale $M_{Z}$

$$
\begin{aligned}
V= & \hat{m}_{1}^{2}\left|H_{1}\right|^{2}+\hat{m}_{2}^{2}\left|H_{2}\right|^{2}-\hat{m}_{3}^{2}\left(\epsilon_{a b} H_{1}^{a} H_{2}^{b}+\text { h.c. }\right) \\
& +\frac{1}{2} \lambda_{1}\left|H_{1}\right|^{4}+\frac{1}{2} \lambda_{2}\left|H_{2}\right|^{4}+\lambda_{3}\left|H_{1}\right|^{2}\left|H_{2}\right|^{2} \\
& +\lambda_{4}\left|\epsilon_{a b} H_{1}^{a} H_{2}^{b}\right|^{2}
\end{aligned}
$$

in terms of the values taken for the set (2.1) and (2.2). (As usual the boundary values of the parameters at $M_{S U S Y}$ read: $\hat{m}_{1,2}^{2}=m_{1,2}^{2}+\mu^{2}, \quad \hat{m}_{3}^{2}=B \mu$ where $m_{1,2}^{2}$ are the soft Higgs mass parameters, $\mu$ is the superhiggs mixing parameter and $B$ is the corresponding soft breaking parameter, $\epsilon_{12}=+1$ and $\hat{m}_{3}^{2}$ is defined to be positive). However, before we proceed further, it is useful to recall the conventional approach 
to radiative $S U(2) \times U(1)$ breaking. It makes use of the renormalization group improved tree-level potential evaluated at a renormalization scale $Q \sim M_{Z}$. The running parameters in the potential, which are functions of the renormalization point $Q$, can be evaluated for any $Q$ by solving the standard supersymmetric RG equations (written in the Landau gauge and in the (mass-independent) modified minimal subtraction scheme, $\overline{M S}$ ) with some universal boundary conditions at the scale $M_{X}$. For $Q \sim M_{Z}$, those RG equations sum up all the large logarithmic corrections proportional to $\ln \left(M_{X} / Q\right)$, which are reabsorbed in the running parameters of the potential (2.10). Due to the supersymmetric structure of the RG equations the tree-level potential maintains its supersymmetric form at any $Q$. Therefore, also at $M_{Z}$, the parameters satisfy the supersymmetric relations:

$$
\lambda_{1}=\lambda_{2}=\frac{1}{4}\left(g_{1}^{2}+g_{2}^{2}\right), \quad \lambda_{3}=\frac{1}{4}\left(g_{2}^{2}-g_{1}^{2}\right), \quad \lambda_{4}=-\frac{1}{2} g_{2}^{2}
$$

This procedure neglects radiative corrections generated by the soft supersymmetry breaking (i.e. neglects logarithmic corrections of the type $\left.\ln \left(m_{\text {stop }} / Q\right)\right)$, which are proportional to large Yukawa couplings and as we know now, can be very important and are of our concern here. One of the well known consequences is that the minimum of the tree-level potential is very sensitive to the choice of the renormalization scale $Q$. The choice $Q=M_{Z}$ gives, in general, unreliable results. The reason is that the loop correction $\Delta V_{1}\left(M_{Z}\right)$ to the potential is not necessarily small. This has been demonstrated explicitly ${ }^{[4]}$ : the sum $V_{o}(Q)+\Delta V_{1}(Q)$ is indeed scale-independent, up to 2-loop corrections which turn out to be small. For the sake of easy reference we give in the Appendix the 1-loop corrections to the parameters of (2.10): $\Delta m_{i}^{2}(Q)$ and $\Delta \lambda_{i}(Q)$ (calculated in the symmetric phase ${ }^{[6]}$ i.e. before $S U(2) \times U(1)$ breaking) generated by the squark loops. This is the dominant part of the entire 1-loop contribution to the effective potential.

For our purpose, the inconvenience of this approach is that one cannot work directly with the tree-level potential (2.10) and therefore the relations between the physical low energy parameters and the unification scale initial conditions are complicated. Fortunately, there exists the well-known equivalent method of including radiative corrections in question, which is based on the Appelquist-Carazzone decoupling theorem. This is the tool to absorb in the running parameters of the tree-level potential (2.10) also the corrections generated by the particle-sparticle mass splitting. The main source of large corrections is in this case the top-stop splitting. At this point one should stress that sparticle thresholds contribute differently to different quantities and the dominant corrections to the scalar potential have different origin than the corrections to the gauge couplings. Therefore, to a very good approximation, the running parameters in (2.10) at the scale $M_{Z}$ can be related to their initial values at the unification scale by solving the $R G$ equations in two steps: the supersymmetric equations in the range $\left(M_{S U S Y}, M_{X}\right)$ and the $\mathrm{RG}$ equations with squarks and gluinos decoupled ${ }^{[6]}$ at the common scale $M_{S U S Y}$, in the

\footnotetext{
${ }^{3}$ The correct choice of the scale $Q$ is such that $\Delta V_{1}(Q) \approx 0$ but it is unknown a priori.
} 
range $\left(M_{Z}, M_{S U S Y}\right)$.f By decoupling squarks at $M_{S U S Y}$ chosen to be equal to the low energy squark soft mass parameters one reabsorbes the logarithmic parts of the corrections (A.7) to (A.16) (in the approximation $m_{Q}^{2}=m_{U}^{2}=m_{D}^{2}$ ) and, of course, also higher order leading logarithmic corrections, into the running parameters of the potential (2.10). As long as we work in the region $\left(M_{Z}, M_{S U S Y}\right)$ with the 1-loop $\mathrm{RG}$ equations, this is a consistent approximation: all non-logarithmic corrections in eqs. (A.7-A.16) are formally the effects of the same order as the neglected 2-loop effects in the RG equations. However, earlier results ${ }^{[4]}$ strongly indicate that the former are much more important than the latter. In particular, as discussed earlier, they are important for the potential to be renormalization scale independent (in our case, this means, independent of the choice of $M_{S U S Y}$ in a reasonable range around the soft squark masses). Also, it is known that the 2-loop effects in the RG equations for the evolution of the quartic couplings are very small ${ }^{[12]}$. Therefore, in this paper we include also the non-logarithmic part of the 1-loop corrections (A.7-A.16) by properly choosing the matching conditions for the parameters $m_{i}^{2}$ and $\lambda_{i}$ at $M_{S U S Y}$. More specifically, we fix the scale $M_{S U S Y}$ so that the contribution (A.8a) to $\Delta m_{2}^{2}$ vanishes. All 1-loop corrections to the quartic couplings are then included by replacing at $M_{S U S Y}$ the continuous matching conditions given by the relations (2.11) by the conditions $\lambda_{i} \rightarrow \lambda_{i}+\Delta \lambda_{i}$ with $\Delta \lambda_{i}$ 's given by the eqs. (A.13A.16) (everywhere in this paper we use the approximation $m_{U_{1,2}}^{2}=m_{Q_{1,2}}^{2}=m_{D_{1,2}}^{2}=$ $m_{D_{3}}^{2}=2 m_{Q_{3}}^{2}-m_{U_{3}}^{2}$ which follows from the supersymmetric RG equations after neglecting terms proportional to the electroweak gauge couplings). Similarly for $m_{i}^{2}, i=1,2,3$, we take discontinuous matching conditions at $M_{S U S Y}: m_{i}^{2}+\Delta m_{i}^{2}$ where $\Delta \hat{m}_{3}^{2}$ is given by the eq. (A.9), $\Delta m_{2}^{2}-$ by the (A.8b) and $\Delta m_{1}^{2}$ is given by the expression (A.7b), always with $\Lambda=M_{S U S Y}$. The technical way of implementing the discontinuous matching conditions for $m_{i}^{2}$ 's is discussed after eq. (2.17).

After the decoupling of the sparticles the largest 1-loop corrections to the tree level potential obtained after the evolution are given by the top quark loops. The top quark should therefore be decoupled at $\Lambda_{\text {top }}$ chosen to minimize those 1 -loop corrections to the vevs $v_{i}$ :

$$
\left.\frac{\partial \Delta V_{1}}{\partial v_{i}}\right|_{v_{i}=v_{i}^{o}}=0
$$

where

$$
\Delta V_{1}=\frac{3}{(4 \pi)^{2}} Y_{t}^{2} v_{2}^{4}\left(-\frac{3}{2}+\ln \frac{Y_{t}^{2} v_{2}^{4}}{\Lambda_{t o p}^{2}}\right)
$$

\footnotetext{
${ }^{4}$ The mass difference between the third and the remaining two generations of squarks can be neglected as the latter contribute little, due to the small Yukawa couplings. Gluino mass is, in most cases, close to the squark masses. The other sparticles are kept in the spectrum down to $M_{Z}$. The reason for using in this paper the RG equations with only squarks and gluinos decoupled is that, on the one side, radiative corrections to the scalar potential from the other sparticles are very small and, on the other side, we want to analyze the light sparticle spectrum predicted by the model. However, as far the scalar potential is concerned, very similar results are obtained by using below $M_{S U S Y}$ the RG equations of the standard model with two Higgs doublets.

${ }^{5}$ We neglect the part (A.7a) of $\Delta m_{1}^{2}$ which is very small for our choice of $M_{S U S Y}$. One should also remember that always $m_{1}^{2} \gg m_{2}^{2}$ and the correction $\Delta m_{1}^{2}$ is relatively much less important than $\Delta m_{2}^{2}$.
} 
We get then $\Lambda_{\text {top }} \sim M_{t} / \sqrt{e}$ and for the interesting range of the top quark masses we can use the approximation $\Lambda_{t o p} \approx M_{Z}$. Thus, by the RG evolution in the range $\left(M_{Z}, M_{S U S Y}\right)$ one reabsorbes in the tree level parameters the remaining large corrections to the potential.

\subsection{Bottom-up approach}

The procedures outlined above are used at two different stages of our approach. First, we come back to the problem of fixing all the parameters in the Higgs potential (2.10) at the scale $M_{Z}$ in terms of the values taken for the set (2.1) and (2.2) and use this procedure to obtain the couplings $\lambda_{i}\left(M_{Z}\right)$ from their boundary conditions $(2.11)$ at $M_{S U S Y}$. In general $\lambda_{i}\left(M_{Z}\right)=\lambda_{i}\left(M_{t}, M_{\tau}, M_{S U S Y}, \tan \beta, g_{k},\left(M_{X}\right)\right)$ as their RG evolution depends on the Yukawa couplings. Given the values of $\lambda_{i}\left(M_{Z}\right)$ we obtain the $\hat{m}_{i}^{2}\left(M_{Z}\right), i=1,2,3$, in terms of $M_{Z}, \tan \beta$ and $M_{A}\left(M_{Z}\right)$ by minimizing the potential (2.10)』. The relevant formulae can be found eg. in ref.[13] and, for easy reference, are collected in the Appendix A.

The second stage of our approach consists in finding the soft supersymmetry breaking parameters $m_{o}, M_{o}, A_{o}, B_{o}$ and the parameter $\mu_{o}$ at the $M_{X}$ which give radiatively induced spontaneous gauge symmetry breaking with the chosen set of values for the low energy observables (2.1) and (2.2). To this end, we notice that in both regions $\left(M_{Z}, M_{S U S Y}\right)^{[20]}$ and $\left(M_{S U S Y}, M_{X}\right)^{[6]}$ the RG equations for the soft Higgs and gaugino mass parameters as well as for the trilinear couplings $A_{i}$ 's can be schematically written as follows $\left(t=\frac{1}{(4 \pi)^{2}} \ln \frac{M_{X}^{2}}{Q^{2}}\right)$ :

$$
\begin{aligned}
& \frac{d}{d t} A_{k}=C_{A A}^{i k} A_{i}+C_{A M}^{i k} M_{i} \\
& \frac{d}{d t} M_{k}=C_{M M}^{i k} M_{i} \\
& \frac{d}{d t} m_{k}^{2}=C_{m m}^{i k} m_{i}^{2}+C_{m M}^{i k} M_{i}^{2}+C_{m A}^{i k} A_{i}^{2}+C_{m \mu}^{k} \mu^{2} \quad k=1,2, Q, U \ldots \\
& \frac{d}{d t} \mu=C_{\mu \mu} \mu
\end{aligned}
$$

(The equation for $\hat{m}_{3}^{2}$ will be discussed later. Note that the parameter $B$ appears only in the equation for $\hat{m}_{3}^{2}$.). The coefficients $C^{i k}$ in eqs.(2.12) depend on the gauge and Yukawa couplings and change at $M_{S U S Y}$ (below $M_{S U S Y}$ they also depend on the $\lambda_{i}$ couplings). Defining the multidimensional vector

$$
\bar{X}=\left(M_{i} M_{j}, M_{i} A_{j}, A_{i} A_{j}, m_{i}^{2}, \mu^{2}\right)
$$

(where eg. $A_{i} A_{j}$ denote all possible products of $A_{i}$ 's present in the model and similarly for the other quantities) and using eqs. (2.12) one has

$$
\frac{d}{d t} \bar{X}(t)=\hat{C}(t) \bar{X}(t)
$$

\footnotetext{
${ }^{6}$ We neglect the running of the pseudoscalar mass $M_{A}$, i.e. we work in the approximation $M_{A}\left(M_{Z}\right)=M_{A}\left(M_{A}\right)$ which is correct to at least few GeV accuracy.
} 
(where $\hat{C}(t)$ is a linear operator) i.e. we have a set of linear, homogeneous equations. Its solution reads:

$$
\begin{array}{cc} 
& \bar{X}(t)=\hat{R}\left(t, t_{o}\right) \bar{X}_{o}\left(t_{o}\right) \\
\text { with } & \hat{R}\left(t, t_{o}\right)=T \exp \left\{\int_{t_{o}}^{t} \hat{C}(s) d s\right\}
\end{array}
$$

where $T$ means chronological ordering in case of non-commuting operators $\hat{C}(t)$. In view of the boundary conditions at $M_{X}$ :

$$
A_{i}^{o}=A_{o}, \quad M_{i}^{o}=M_{o}, \quad m_{i}^{o}=m_{o}
$$

the solution (2.14) can be written more explicitly as 1 (we recall that $\hat{m}_{i}^{2}=m_{i}^{2}+$ $\left.\mu^{2}, \quad i=1,2\right)$.

$$
\hat{m}_{i}^{2}\left(M_{Z}\right)=c_{i j}\left(M_{t}, M_{\tau}, g_{k}^{2}, \tan \beta, M_{S U S Y}\right) P_{j}^{o}, \quad i=1,2
$$

where

$$
P^{o}=\left(M_{o}^{2}, m_{o}^{2}, \mu_{o}^{2}, A_{o}^{2}, M_{o} A_{o}\right) .
$$

Quite similarly one gets

$$
m_{Q, U}^{2}\left(M_{S U S Y}\right)=b_{Q, U}^{j}\left(M_{t}, M_{\tau}, g_{k}^{2}, \tan \beta, M_{S U S Y}\right) P_{j}^{o}
$$

The coefficients $c_{i j}$ and $b_{Q, U}^{j}$ in the algebraic equations (2.15) and (2.16) are found by integrating numerically the RG equations with the boundary conditions like $P_{1}^{o}=1, \quad P_{i}^{o}=0$ for $i \neq 1$ etc. This is a two-step procedure: first, the supersymmetric equations are integrated from $M_{X}$ to $M_{S U S Y}$ with those boundary conditions at $M_{X}$. Then the RG equations with decoupled squarks and gluinos are integrated from $M_{S U S Y}$ to $M_{Z}$, with the boundary values at $M_{S U S Y}$ obtained from the first integration. $M_{S U S Y}$ is fixed in advance by the choice of the squark masses, eq. (2.2).

Thus, we have a set of 4 algebraic equations for 4 independent parameters $M_{o}, m_{o}, \mu_{o}, A_{o}$. Finally, $B_{o}$ is fixed from

$$
\hat{m}_{3}^{2}\left(M_{S U S Y}\right)=\mu\left(M_{S U S Y}\right) B\left(M_{S U S Y}\right)
$$

by the equation

$$
\hat{m}_{3}^{2}\left(M_{S U S Y}\right)=d_{1} \mu_{o} M_{o}+d_{2} \mu_{o} A_{o}+d_{3} \mu_{o} B_{o}
$$

(as before, the coefficients $d_{i}$ are calculated from the RG equations).

At this point we can complete our discussion of implementing the matching conditions for $\Delta m_{i}^{2}$ at $M_{S U S Y}$. All the terms (A.7b), (A.8b) and (A.9) are proportional to the parameters $\mu$ and $A_{i}$ taken at $M_{S U S Y}$. It follows from the eqs. (2.12) that, analogously to (2.15),

$$
\begin{aligned}
& \mu=c \mu_{o} \\
& A_{i}=c_{A}^{i} A_{o}+c_{M}^{i} M_{o}
\end{aligned}
$$

\footnotetext{
${ }^{7}$ Analogous equations have been discussed earlier in ref.[14] with the coefficients derived analytically (in the case of no sparticle decoupling and negligible $Y_{b}$ ).
} 
and, therefore, the corrections $\Delta m_{i}^{2}$ have identical structure in terms of the unifica-

tion scale parameters as the $m_{i}^{2}$ 's themselves. So, their inclusion amounts to some modification of the coefficients $c_{i j}$ and $b_{Q, U}^{j}$ in the eq. (2.15) and (2.16).

Our procedure is now completed and can be summarized as follows: for any chosen set of values for the physical parameters (2.2) we find the corresponding low energy parameters of the effective lagrangian and then solve the algebraic equations (2.15), (2.16) and (2.17) to find the lagrangian parameters at $M_{X}$ which give those chosen values of $\tan \beta, M_{A}, m_{Q}^{2}$ and $m_{U}^{2}$. We note that for each set (2.2) there are up to 8 solutions for the parameters at $M_{X}$ : the sign of $m_{o}$ is taken to be positive by convention (the lagrangian is symmetric under an overall change of sign of all soft breaking terms) but the solutions appear in pairs of $\pm M_{o}$ and $\pm \mu_{o}$ (the solutions e.g. for $M_{o}<0$ are the same as the solutions for $M_{o}>0$ and the reversed signs of all parameters, with exception of $m_{o}$ ). The remaining two-fold ambiguity follows from the fact that the set $(2.15-2.16)$ is equivalent to a quadratic equation for the ratio $A_{o} / M_{o}$. This overall eight-fold ambiguity is simply a reflection of the fact that the set (2.2) is not a complete set of observables in the model: different solutions give different prediction for other observables. Finally, one should stress that for a given set of values for the observables (2.2) our equations may have no solution at all. This is, in fact, the most often encountered case for a random choice of the values (2.2) and it simply means that the given set cannot be obtained in the model. Our procedure in this paper is to fix $M_{t}$ and $\tan \beta$ and then to scan systematically the values of $M_{A}, m_{Q}^{2}$ and $m_{U}^{2}$ in the range, say, from 0 to $1 \mathrm{TeV}$, by varying them in steps of a few $\mathrm{GeV}$ in search for solutions to our equations. Thus, for a given choice of $M_{t}$ and $\tan \beta$ we get a range of values for $M_{A}, m_{Q}^{2}$ and $m_{U}^{2}$ which are obtainable in the model (i.e. for which the solutions to eqs. (2.15) and (2.16) exist). For each solution we can then calculate the values of the other physical observables. Finally, we impose on the obtained solutions the "no fine tuning" constraint and all the presently available experimental constraints.

\section{Qualitative Features of the Solutions}

In this section we discuss those properties of our solutions which follow from the requirement of radiatively induced gauge symmetry breaking alone. We also include the constraint of all masses squared to be positive and the usual one of no deeper lying minima of the scalar potential that break charge and/or colour[15]: $A_{t}^{2}<3\left(m_{Q}^{2}+m_{U}^{2}+\hat{m}_{2}^{2}\right)$ and similarly for the remaining $A_{i}$ 's at low energy, as well as the analogous condition at the unification scale. Finally, we demand that the scalar potential should be bounded from below at the scale $M_{X}$.

The "naturalness" constraint and the constraints from the present experimental limits will be discussed in the next sections. As an introduction to our results let us recall certain properties of the low energy potential (2.10). One can verify that radiative corrections stabilize the potential ${ }^{[6,14]}$. So, the low energy mass parameters $\hat{m}_{i}, \quad i=1,2,3$, which correspond to the chosen values of $\tan \beta$ and $M_{A}$, do not need, in general, to satisfy at $M_{Z}$ the well known stability condition for the supersymmetric tree level potential: $\hat{m}_{3}^{4}<\left(\hat{m}_{1}^{2}+\hat{m}_{2}^{2}\right)^{2} / 4$. Secondly, as already mentioned earlier, up to the standard model 1-loop corrections, the effective potential is now 
renormalization scale independent in the range $\left(M_{Z}, M_{S U S Y}\right)$. This means that the gauge symmetry is broken at the tree level already at $M_{S U S Y}$, i.e. at the scale where the quartic couplings in the potential (2.10) recover their supersymmetric values. 8 Note, however, that the individual parameters in the potential and in the lagrangian, in general, run sizably in the range $M_{Z}, M_{S U S Y}$ (particularly the quartic scalar couplings !). Physical quantities at the scale $M_{Z}$ receive in addition the SM 1loop corrections (particularly, from the top quark and vector boson exchange) which are included into the analysis by our choice of the renormalization scale $Q=M_{Z}$.

Next, it is instructive to analyze the coefficients in eqs.(2.15) and (2.16) and to understand them qualitatively with help of the RG equations. A sample of coefficients for $\hat{m}_{i}^{2}, m_{Q}^{2}$ and $m_{U}^{2}$ is given in Table 1. Of special interest is their dependence on the top quark mass, $\tan \beta$ and their modification by radiative corrections. A remark is in order here: it is the usual procedure followed by many authors to solve the supersymmetric RG analytically in some approximation. We could, of course, follow the same approach here to calculate the coefficients in eq. (2.15) and (2.16). However, the approximations involved in this procedure make it very difficult, if not impossible, to discuss the most interesting, just mentioned, dependences. Therefore, we prefer to base our discussion on numerical results, guided by general qualitative features which can be inferred directly from the RG equations. Let us first look at $\hat{m}_{1}^{2}$ and $\hat{m}_{2}^{2}$ for small $\tan \beta$. Taking into account the definitions $\hat{m}_{i}^{2}=m_{i}^{2}+\mu^{2}$ and the initial conditions $m_{i}^{2}=m_{o}^{2}$, we expect from the RG equations (dominated by the terms proportional to $Y_{t}$ - see the Appendix B) that for $\hat{m}_{1}^{2}$ the coefficients are close to 1 for $m_{o}^{2}$ and $\mu_{o}^{2}$, positive and smaller for $M_{o}^{2}$ and negligible for $A_{o}^{2}$ and $M_{o} A_{o}$. For $\hat{m}_{2}^{2}$ the coefficient of $\mu_{o}^{2}$ is expected again to be $0(1)$ but the coefficient of $m_{o}^{2}$ gets additional negative contribution from the terms proportional to $Y_{t}$ which largely cancels the +1 contribution from the initial condition. The most interesting is the coefficient of $M_{o}^{2}$ which is $0(1)$ and negative! This looks contradictory to the positive gauge terms in the $R G$ equation for $m_{2}^{2}$ (the same as for $m_{1}^{2}$ ) and is the reflection of the running of the scalar masses in the equation for $m_{2}^{2}$. We have

$$
\begin{aligned}
\frac{d}{d t}\left(m_{Q}^{2}, m_{U}^{2}\right) & =\frac{16}{3} g_{3}^{2} M_{3}^{2}+\ldots \\
\frac{d}{d t}\left(M_{3} / g_{3}^{2}\right) & =0, \quad M_{3}\left(M_{X}\right)=M_{o}
\end{aligned}
$$

and this strong dependence on $M_{o}^{2}$ is transmitted to $m_{2}^{2}$ through the negative term $-3 Y_{t}\left(m_{Q}^{2}+m_{U}^{2}+\ldots\right)$ in the equation for $m_{2}^{2}$. Naively a second order effect, it is in fact the dominant one due to the large logarithmic enhancements (and the running of $M_{3}$ ) and it plays an important role in understanding our results. In particular it explains strong dependence of the $M_{o}^{2}$ coefficient in $m_{2}^{2}$ on $M_{S U S Y}$ : the larger is this scale the smaller is the absolute value of the negative $M_{o}^{2}$ coefficient, due to the earlier decoupling of the gluino term in eq.(3.1). This behaviour has also important implications for the fine tuning problem (to be discussed in the next section) before and after radiative corrections. Indeed, in our formalism, neglecting

\footnotetext{
${ }^{8}$ Another way of seeing this is to remember that the driving force for the difference in the $m_{1}^{2}$ and $m_{2}^{2} \mathrm{RG}$ evolution from the scale $M_{X}$ (which gives the breaking at low energy) are squarks and they are decoupled at $M_{S U S Y}$.
} 
radiative corrections to the scalar potential means setting $M_{S U S Y}=M_{Z}$ for any values of the stop mass parameters and we see (the numbers in brackets in Table 1) that the $M_{o}^{2}$ coefficients in $m_{2}^{2}$ are then, for the same set of values for the variables (2.2), much larger in the absolute values than after radiative corrections.

It is also very important to recall that the low energy values of $m_{Q}^{2}$ and $m_{U}^{2}$ are almost directly given by the values of $M_{o}^{2}$. The next-to-leading dependence is on $m_{o}^{2}$ but with the coefficients by factor $\mathrm{O}(10)$ smaller then for $M_{o}^{2}$. Again, the $M_{o}^{2}$ coefficients decrease substantially after radiative corrections.

The $\tan \beta$ and $M_{t}$ dependence of the coefficients is mainly determined by the behaviour of the Yukawa couplings and their contribution to the RG equations. For fixed $M_{t}$ and increasing $\tan \beta$ the $Y_{b}$ becomes larger and the $Y_{t}$-smaller (eventually, $Y_{t} / Y_{b} \cong 1$ for $\left.\tan \beta \cong m_{t}\left(M_{t}\right) / m_{b}\left(M_{t}\right)\right)$. Accordingly, the coefficients in the equation for $\hat{m}_{1}^{2}$ behave as follows: the $M_{o}^{2}$ coefficient is driven from positive to negative values, the one for the $m_{o}^{2}$ is also decreasing but remains positive, due to the $m_{o}^{2}$ contribution to $\hat{m}_{1}^{2}$, and the $M_{o} A_{o}$ coefficient is slowly rising from zero to $\sim 0.4$. This behaviour follows from the increase of $Y_{b}$ which starts to play the same role for $\hat{m}_{1}^{2}$ as $Y_{t}$ for $\hat{m}_{2}^{2}$. At the same time the $Y_{t}$ is decreasing (but it changes much less than $Y_{b}$ ) and, in consequence, the coefficients in the equation for $\hat{m}_{2}^{2}$ change just in the opposite direction. Still, even for $\tan \beta \approx m_{t} / m_{b}$ the coefficients remain different enough from those in the expression for $\hat{m}_{1}^{2}$ to assure the existence of solutions ${ }^{[16]}$. The dependence on $M_{t}$ (for fixed $\tan \beta$ ) can be easily understood in a similar way, and follows from changing $Y_{t}$. (We note also the decrease of the $\mu_{o}^{2}$ coefficients with increasing $Y_{t}$ ).

Generically, the mass parameters of the low energy potential satisfy the relation $\hat{m}_{1}^{2} \gg \hat{m}_{2}^{2}$ and $\hat{m}_{2}^{2} \leq 0$. Thus, to understand qualitatively the pattern of our solutions, in the first approximation we can take $m_{2}^{2}=0$. In a large region of the parameter space we then have:

$$
\begin{aligned}
& \hat{m}_{1}^{2} \approx a M_{o}^{2}+b m_{o}^{2}+c \mu_{o}^{2} \\
& \hat{m}_{2}^{2} \approx-\alpha M_{o}^{2}+\beta m_{o}^{2}+\gamma \mu_{o}^{2} \approx 0
\end{aligned}
$$

with $b, c, \alpha, \gamma$ positive and $a$ and $\beta$ generally smaller and of both signs. This structure, together with the relation $M_{A} \approx m_{1}^{2}$ and

$$
m_{\tilde{q}}^{2} \approx C_{\tilde{q}}\left(M_{o}^{2}+O\left(\frac{1}{10}\right) m_{o}^{2}\right)
$$

explains the correlations seen in our solutions for the absolute values of the pairs $\left(M_{o}, \mu_{o}\right),\left(M_{o}, m_{o}\right)$ and $\left(\mu_{o}, m_{o}\right)$. It is most visible in the $\left(M_{o}, \mu_{o}\right)$ correlation (or, equivalently, $\left(M_{2}, \mu\right)$ ) which is interesting in the context of the neutralino properties predicted by the model. It is clear from the coefficients in Table 1 that, as long as we can neglect the term $M_{o} A_{o}$ in the equation for $\hat{m}_{2}^{2}$ (and neglecting also the other small coefficients), crudely speaking, the solutions exhibit approximately linear correlation $M_{o}^{2} \approx(\gamma / \alpha) \mu_{o}^{2}$. The actual allowed regions in the space $\left(M_{2}, \mu\right)$ are determined by the integration over the soft scalar masses $m_{Q}^{2}$ and $m_{U}^{2}$, and over the pseudoscalar mass $M_{A}$. They are shown in Fig. 5 for $\tan \beta=2$ and 10 for $M_{t}=130 \mathrm{GeV}$ and for $\tan \beta=2$ and 30 for $M_{t}=180 \mathrm{GeV}$. A remark 
has to be made here. In this section we refer to all the solutions shown in the Figures. The distinction between experimentally excluded or allowed and natural or unnatural solutions (which is marked in the Figures) will be discussed in the subsequent sections. The dependence of the $\left(M_{2}, \mu\right)$ correlation on $\tan \beta$ and $M_{t}$ follows directly from the discussed above properties of the coefficients and in the first, crude, approximation is just the reflection of the changing slope $a$ in $M_{o}^{2} \approx a \mu_{o}^{2}$. The correlation $\left(M_{2}, \mu\right)$ has direct consequence for the predicted neutralino properties. It is well known ${ }^{[17]}$, and follows directly from the structure of the gaugino-higgsino mass matrix, that in the limit of large $M_{2} \gg \mu$ the lightest neutralino becomes almost pure higgsino with $M_{n}=|\mu|$ and in the limit of large $\mu \gg M_{2}$ - pure gaugino with $M_{n} \approx M_{2} / 2$. The pattern observed in the $\left(M_{2}, \mu\right)$ plane explains qualitatively the results shown in Fig. 7 . We plot there the gaugino content $Z_{n}=$ $Z^{2}(1,1)+Z^{2}(1,2)$ of the lightest neutralino as a function of the neutralino mass, as predicted by our solutions. (As usual we define $\chi_{1}=Z(1,1) \hat{B}+Z(1,2) \hat{W}^{3}+$ $Z(1,3) \hat{H}_{1}+Z(1,4) \hat{H}_{2}$ where $Z(i, j)$ is the real orthogonal matrix that diagonalizes the neutralino mass matrix.) In particular, the $M_{t}$ and $\tan \beta$ dependence of those results is easily interpretable in terms of the analogous dependence of the $\left(M_{2}, \mu\right)$ correlation.

The above simple discussion breaks down and a new feature of our solutions becomes visible for the values of $M_{t}$ and $\tan \beta$ such that the coefficient of the $M_{o}^{2}$ term in the equation for $\hat{m}_{1}^{2}$ is much smaller in its absolute value than that of the $M_{o} A_{o}$ term. For instance, this is the case for $M_{t}=160 \mathrm{GeV}$ and $\tan \beta=20$. Then the dependence on $M_{o}$ of the $\hat{m}_{1}^{2}$ comes from the $M_{o} A_{o}$ term and is correlated with the values of $A_{o}$. Positive $A_{o}$ allows for larger positive $M_{o}$, since then the $M_{o} A_{o}$ term in $\hat{m}_{2}^{2}$ (no longer negligible) is positive and helps to cancel out the negative $M_{o}^{2}$ term. Neutralinos with large gaugino content (large $\mu$, small $M_{2}$ ) appear mainly for light $m_{Q}^{2}$ (hence small $M_{o}$ and $\left(-|A|_{o}\right)$ branch) and large $M_{A} \approx \hat{m}_{1}^{2}$ (hence large $\mu$ ). For even larger values of $M_{t}$ and $\tan \beta$ (e.g. $M_{t}=180 \mathrm{GeV}, \tan \beta=30$ ) the coefficient of the $M_{o}^{2}$ term in the equation for $\hat{m}_{1}^{2}$ becomes again large in the absolute value (but negative) and the effect disappears.

Another interesting correlation present in the model is seen in the $\left(m_{\tilde{t}}, M_{o}\right)$ plane $\left(m_{\tilde{t}}\right.$ is the mass of the lightest squark), as illustrated in Fig. 10. It follows from the structure of eq. (3.3) and from the qualitative features of the coefficients shown in Table 1: the $m_{\tilde{t}}$ is almost directly related to $M_{o}$ 甲. One can also expect some correlation in the $\left(m_{\tilde{t}}, M_{A}\right)$ plane. As explained above, $M_{o}$ is correlated with $\mu_{o}$ (up to the contribution of the $m_{o}$ and $A_{o}$ terms) and therefore $M_{A}^{2} \cong \hat{m}_{1}^{2}$ should be correlated with $m_{\tilde{t}}$. However, as seen from Fig. 12, the dependence on the additional parameters makes the final correlation rather weak. An obvious correlation exists between $m_{\tau}$ and the $m_{o}$, Fig. 11. The qualitative difference between Fig. 11A, $\mathrm{C}$ and Fig. $11 \mathrm{~B}, \mathrm{D}$ is due to the negative contribution of the $Y_{b}$ term to the RG equation for $m_{\tilde{\tau}}$, which is increasing for large $\tan \beta$.

In Fig.1-4 we also show our solutions in the planes $\left(m_{o}, M_{o}\right),\left(m_{o}, A_{o}\right),\left(A_{o}, M_{o}\right)$ and $\left(A_{o} / m_{o}, B_{o} / m_{o}\right)$. Clear positive correlation is observed in the $\left(m_{o}, A_{o}\right)$ projec-

\footnotetext{
${ }^{9}$ Strinctly speaking, this is true for the soft squark masses. However, the results obtained after diagonalization of the stop mass matrix (presented in Fig. 10), with the mixing terms predicted by our solutions, show very similar pattern.
} 
tion. Interesting structure is seen in the $\left(A_{o} / m_{o}, B_{o} / m_{o}\right)$ plane: there is an almost linear correlation (see eq. (2.17) and Table 1) and two branches of solutions exist, corresponding to the two solution with $\mu= \pm|\mu|$, and with the gap depending on the value of $\hat{m}_{3}^{2}$. Only weak correlation is observed in the $\left(m_{o}, M_{o}\right)$ plane. Obviously, it will be very interesting to study correlations in 3 and more variables.

So far we have been discussing the qualitative features of the predictions based on the solutions to eqs.(2.15), (2.16) and (2.17), i.e. following from the requirement of radiatively induced gauge symmetry breaking. In the next two sections we impose two additional constraints: no fine tuning and the present experimental limits.

\section{Fine Tuning}

The basic feature of the model is to predict the low energy parameters in terms of the unification scale parameters which, hopefully, can be obtained in future from some underlying theory. This procedure involves extrapolations of many orders of magnitude in the energy scale. In this context it is important to address the question of the degree of fine tuning of the parameters at $M_{X}$ which is necessary to get the correct low energy results. Precise values of the input parameters do not, by themselves, mean fine tuning yet. Only when the precision we need for the input parameters is much higher than the precision we require for the low energy results, it means fine tuning. In other words it is the question of response in the low energy results $Q_{i}$ to small changes in the high energy input $P_{j}$, which is measured by ${ }^{[2]}$

$$
\Delta_{i j}=\frac{\delta Q_{i}}{Q_{i}} / \frac{\delta P_{j}}{P_{i}}
$$

Equivalently this is also a measure of cancellations in equations like (2.15): for instance, calculating the mass parameters $\hat{m}_{i}^{2}$ in the low energy lagrangian in terms of the high energy parameters $P_{i}$ (using the coefficients like those in Table 1) the single terms on the rhs should not be much larger than the calculated masses. To some extent it is a matter of taste how big cancellations (i.e. how big $\Delta_{i j}$ 's) are still tolerable. The coefficients $c_{i j}$ in eqs. (2.15)-(2.17) depend on large logaritms $\ln M_{X} / M_{Z} \cong 0(30)$, so we take here the attitude that cancellations of this order of magnitude are still "natural".

Our actual interest is to avoid fine tuning when calculating the physical quantities like $M_{Z}$ (or $v$ ), $\tan \beta, M_{t}$, etc. So we look for solutions with acceptable all the following derivatives

$$
\begin{aligned}
\Delta_{i j}=\frac{P_{i}}{Q_{j}} \frac{\partial Q_{j}}{\partial P_{i}} \quad, \quad Q_{i} \equiv\left\{Y_{t}, Y_{\tau}, v, \tan \beta, M_{A}, m_{Q}, m_{U}\right\} \\
P_{i} \equiv\left\{Y_{t}^{o}, Y_{\tau}^{o}, M_{o}, m_{o}, \mu_{o}, A_{o}, B_{o}\right\}
\end{aligned}
$$

We shall consider a solution to be "natural" if

$$
\left|\Delta_{i j}\right|<A, \text { for } i, j=1, \ldots 7
$$

where, following the arguments given above, we sort the solutions according to $A=10,30$ and no constraint on $\left|\Delta_{i j}\right|$. It turns out, however, that in the range of 
the low energy parameters studied in this paper, $M_{A}, m_{Q}, m_{U}<1 \mathrm{TeV}$ almost all solutions satisfy $\left|\Delta_{i j}\right|<100$ (for moderate values of $\tan \beta$ discussed here).

For given values of $v$ and $\tan \beta$ the constraints (4.3) mean upper limits on the unification scale parameters, or equivalently, upper limits on $M_{A}$ and the stop masses for which "natural" solutions exists. In general, it is difficult to avoid large cancellations simultaneously in all the low energy mass parameters.

There are two main effects which ease the problem of fine tuning after radiative corrections. These are: increase of the absolute value of $\hat{m}_{2}^{2}$ for the same set of low energy parameters (see eq. (A.4) and remember that, generically $\hat{m}_{2}^{2} \leq 0$ ) and (the discussed earlier) decrease of some coefficients in eqs.(2.15)-(2.17), particularly the ones of the $M_{o}^{2}$ terms. The overall effect is that the maximal acceptable $M_{2}$ considered as a function of the $\max \left|\Delta_{i j}\right|$ is, with radiative corrections included, almost twice as large as without. Similar effect exists for $m_{\tilde{t}}$ and $\mu$. This results in a sizable enlarging of the "natural" regions (for any chosen value of $A$ ) on all the Figures. For the illustration of this point we mark in Figs, 1, 5, 7, 12 (by the solid contours) the "natural" regions, for $A=30$, obtained without radiative corrections. One should also remark that for $A=10$ the allowed parameter space of the model is small even with radiative corrections included. The fine tuning problem becomes more severe for $\tan \beta$ close to 1 and to $m_{t} / m_{b}$, i.e. close to the limits of the $\tan \beta$ range obtainable in this model. Solutions for the large $\tan \beta$ case will be discussed in more detail in Section 6.

A comment is in order here on our method of calculating derivatives $\partial Q_{i} / \partial P_{j}$ with $P_{k}$ fixed, for $k \neq j$. Note that the coefficients $c_{i j}$ in eqs.(2.15)-(2.16) are fixed for given values of the low energy parameters (in particular they depend on the decoupling point which is fixed in terms of the low energy squark mass parameters). Thus, it is straightforward for us to calculate the inverse matrix $\partial P_{j} / \partial Q_{i}, Q_{k}$ fixed for, $k \neq i$, and to find by inversion the matrix of interest. The direct calculation of the latter is in our approach cumbersome because fixing the unification scale parameters rather then the low energy parameters makes the coefficient $c_{i j}$ dependent on them (the decoupling point depends then on $P_{i}$ 's). Thus, we calculate:

$$
\left.\frac{\partial Q_{i}}{\partial P_{j}}\right|_{P_{i \neq j}}=\left[\left.\frac{\partial P_{k}}{\partial Q_{l}}\right|_{Q_{m \neq l}}\right]^{-1}
$$

Note, that to get the correct answer for the lhs derivatives one has to calculate the complete matrix on the rhs. Most often, the largest derivatives are those of the $v$ with respect to $Y_{t}, \mu_{o}$ and $A_{o}$ and of $\tan \beta$ with respect to $B_{o}, m_{o}, A_{o}$ and $Y_{t}$. Finally, one should remark that in eq. (4.2) we consider only the parameters which are used in the calculation as independent input parameters. It also happens that the derivatives of some other physical quantities (e.g. sparticle masses), taken with respect to the high energy set $P_{i}$, become large, even when $\Delta_{i j}$ 's defined in eq. (4.2) are small. 


\section{Experimental Constraints and Final Results}

Let us recapitulate our procedure: For some chosen values of the low energy physical parameters $M_{t}, \tan \beta, M_{A}, m_{Q}$ and $m_{U}$ (see eq. (2.2)) we look for solutions to eqs. (2.15)- (2.17). These are the equations for the unification scale parameters, derived from the requirement of radiatively induced gauge symmetry breaking in the minimal supergravity model. The existence (non-existence) of solutions for a given set of input values means that this particular set can (cannot) be realized in the model. In this paper we present results for a few generic top mass and $\tan \beta$ values: $M_{t}=130 \mathrm{GeV}$ and $\tan \beta=2$ and $10 ; M_{t}=180 \mathrm{GeV}$ and $\tan \beta=2$ and 30. In the next Section we discuss very large $\tan \beta$ cases: $\tan \beta \approx m_{t} / m_{b}$. In each case the $M_{A}, m_{Q}$ and $m_{U}$ have been scanned in the region from 0 to $1 \mathrm{TeV}$ in steps of a few $\mathrm{GeV}$. Once some solutions for the unification scale parameters are found we can predict the values of any other low energy observables, corresponding in the model to our set of input data. We present our results in form of the scatter plots obtained by our scanning procedure. In this paper we limit ourselves to two-dimensional scatter plots, but multi-dimensional correlations are also of interest. In Fig. 1-4 we present our solutions in the unification scale variables $\left(m_{o}, M_{o}\right), \quad\left(m_{o}, A_{o}\right),\left(A_{o}, M_{o}\right)$ and $\left(B_{o} / m_{o}, A_{o} / m_{o}\right)$. Projections onto the variables $\left(M_{2}, \mu\right),\left(M_{n}, M_{A}\right),\left(Z_{n}, M_{n}\right),\left(M_{c}, M_{A}\right),\left(M_{c}, M_{n}\right),\left(m_{\tilde{t}}, M_{o}\right),\left(m_{\tilde{\tau}}, m_{o}\right)$, $\left(m_{\tilde{t}}, M_{A}\right)$ and $\left(M_{c}, m_{\tilde{t}}\right)$ are shown in Fig. $5-13$. (We recall the notation: $M_{o}, m_{o}, \mu_{o}$, $A_{o}, B_{o}$ are the high scale parameters; $M_{2}$ is the low energy $S U(2)$ gaugino mass, $\mu$ is the low energy supersymmetric Higgs mixing parameter; $M_{A}, M_{n}, M_{c}, m_{\tilde{t}}, m_{\tilde{\tau}}$ are the pseudoscalar, and the lightest neutralino, chargino, stop and stau physical masses, respectively, and $Z_{n}$ is the gaugino content of the lightest neutralino. Three different classes of solutions are introduced: a) solutions already excluded by the present experimental constraints, b) allowed by the present experiments and satisfying the criterion $\max \left|\Delta_{i j}\right|<30$, c) allowed by experiment and with $30<$ $\max \left|\Delta_{i j}\right|<100$. (In the considered region of $M_{A}, m_{Q}, m_{U}<1 \mathrm{TeV}$ and for those moderate values of $\tan \beta$, solutions with $\max \left|\Delta_{i j}\right|>100$ occur quite rarely.) It is worth pointing out that, in our 2-dimensional projections of the 5-dimensional parameter space, those three different classes of solutions are often placed in overlapping regions. For clarity of the Figures, only part of the obtained solutions is plotted: only as many of them as is needed to properly mark various domains in the parameter space. Additional important information given in some of the Figures is the sensitivity of our results to radiative corrections. The solid contours in Fig. 1,5,7,12 encircle the regions where the class (b) solutions are present, with no radiative corrections included. In the same Figures, the dashed contours show the regions where $\max \left|\Delta_{i j}\right|<10$.

For the purpose of this paper we have taken the following "experimental" limits as the constraints for our solutions:

$$
\begin{aligned}
& m_{\tilde{g}}>120 \mathrm{GeV}, m_{\tilde{l}}>45 \mathrm{GeV}, M_{c}>45 \mathrm{GeV} \\
& \left|Z^{2}(1,3)-Z^{2}(1,4)\right|<0.03,|Z(1,3) Z(2,3)-Z(1,4) Z(2,4)|<0.04 \\
& \text { (for } M_{n}<45 \mathrm{GeV} \text { ) (for } M_{n_{1}}+M_{n_{2}}<45 \mathrm{GeV} \text { ) } \\
& m_{\tilde{q}}>100 \mathrm{GeV} \text { (for the first two generations of squarks), }
\end{aligned}
$$




$$
m_{\tilde{t}}>45 \mathrm{GeV}, \quad M_{A}>40 \mathrm{GeV} .
$$

Some of the above limits are somewhat rough representation of the actual experimental data. In particular, strictly speaking, at present there is no absolute lower limit on the stop mass ${ }^{[18]}$. The bounds from $p \bar{p}$ colliders do not apply to it; those bounds assume degenerate squarks and this assumption fails for strong $\mathrm{L}-\mathrm{R}$ mixing of the $\tilde{t}$-squarks. Also searches at the $e^{+} e^{-}$colliders are affected since the $\mathrm{L}-\mathrm{R}$ mixing can reduce the $Z \tilde{t}_{1} \tilde{t}_{1}$ coupling $\square$. In the model considered, each solution gives definite predictions for mass mixings and couplings and in principle a more detailed phenomenological analysis with respect to the constraints from the present $p \bar{p}$ and $e^{+} e^{-}$data is possible for each solution. We postpone it, however, for future study and take (5.1) as sufficient for our discussion here.

As it has already been mentioned several times, the model shows a high degree of correlation among various physical quantities which results from the small number of free parameters. We have discussed in Section 3 that a number of correlations follows from the requirement of radiatively induced gauge symmetry breaking. We can add now to this discussion some remarks following from the fine tuning and the experimental constraints. As expected, the larger the values of the high energy parameters the more fine tuning is required (see Fig. 1-4). For instance, for $\max \left|\Delta_{i j}\right|<30$ we get $M_{o}<(200-400) \mathrm{GeV}$ and $m_{o}<(400-900) \mathrm{GeV}$, depending on the values of the top quark mass and $\tan \beta$. Simultaneously, the ratio $m_{o} / M_{o}$ can vary, approximately, in the range (0.1-10). As we know from Section 3, the gauge symmetry cannot be broken for $m_{o} \approx M_{o} \approx 0$. At least one of them must be visibly different from zero. The experimental constraints put somewhat stronger lower bound on $M_{o}(>70 \mathrm{GeV})$ than on $m_{o}(>(20-30) \mathrm{GeV})$ and acceptable solutions with $m_{o} \approx A_{o} \approx B_{o} \approx 0$ exist.

At the quantitative level, the predictions of the model show strong dependence on the top quark mass, $\tan \beta$ and on the inclusion of the radiative corrections. For a detailed survey of the predictions for the physical (low energy) observables we refer the reader to Fig. 5-12. Here we would like to stress again the interesting correlations between $M_{2}$ and $\mu$, between $M_{t}$ and $Z_{n}$ (the gaugino content of the lightest neutralino), $\tan \beta$ and $Z_{n}, Z_{n}$ and $M_{n}, m_{\tilde{t}}$ and $M_{A}$ etc. Similarly to the requirement of radiative gauge symmetry breaking, the experimental constraints (5.1) have "global" effects, i.e. each individual constraint affects not only this concrete quantity but also all the others through the rigid structure of the model. An interesting example is the interplay of the experimental lower bounds on the gluino, chargino and neutralino masses. In the model the three masses are interconnected by the parameters $M_{2}$ (or $M_{o}$ ), $\mu$ and $\tan \beta$ and it varies from case to case which experimental bound is most operative in fixing e.g. the effective lower bound for the neutralino mass. For neutralinos with large higgsino content ( $\mu$ small, $M_{2}$ large), i.e. with large coupling to the $Z$, the LEP data give directly $M_{n}>45 \mathrm{GeV}$. For gaugino-like neutralinos ( $M_{2}$ small, $\mu$ large) the bound on $M_{n} \approx M_{2} / 2$ follows from the bound on the gluino mass $\left(M_{2} \approx 0.3 m_{\tilde{g}}>40 \mathrm{GeV}\right)$ or from the LEP bound on the chargino mass (for $M_{2} \ll \mu$ and $\mu>M_{W}$ one has $M_{c} \sim M_{2}$ ). More precise numbers follow from the well known complete chargino and neutralino mass matrices

\footnotetext{
${ }^{10}$ Similar remark applies also to stau leptons.
} 
and depend on the actual values of the parameters in the given case. As seen e.g. in Fig. 7, the lower bound for $M_{n}$ is indeed in the range (20-50) GeV, depending on $M_{t}$ and $\tan \beta$.

Another interesting example is the effective lower limit for the pseudoscalar mass $M_{A}$. We have taken $M_{A}>40 \mathrm{GeV}$ as the experimental lower limit for $M_{A}$ but, as seen e.g. in Fig. 6,8 and 12, the effective bound (which follows from the other constraints (5.1)) is much higher, $M_{A}>175,125,205,150 \mathrm{GeV}$ for $\left(M_{t}, \tan \beta\right)=(130,2),(130,10),(180,2)$ and $(180,30)$, respectively. One can understand it as follows: $M_{A}^{2} \approx \hat{m}_{1}^{2}$ and, for the discussed here moderate values of $\tan \beta$, all coefficients in the equation (2.15) for $\hat{m}_{1}^{2}$ are positive and large (see Table 1). Therefore, small $M_{A}^{2}$ implies small values of $M_{o}^{2}, m_{o}^{2}$ and $\mu_{o}^{2}$. However, as discussed above, there are lower bounds on those parameters from the experimental lower bounds on the gluino, neutralino and chargino masses which, as shown in Fig. 6 and 8, give the effective lower bound for $M_{A}$ of the order $\mathrm{O}(200 \mathrm{GeV})$.

For larger values of $M_{t}$ and $\tan \beta$ (e.g. $M_{t}=180 \mathrm{GeV}$ and $\tan \beta=30$ ) the coefficients in $\hat{m}_{1}^{2}$ of the $M_{o}^{2}$ and $\mu_{o}^{2}$ terms become very close to each other and of opposite signs. The above argument does not then work any more: arbitrarily large values of $M_{o}^{2} \approx \mu_{o}^{2}$ can give small $M_{A}$. However, we see that, in the case considered, the coefficients of the $M_{o}^{2}$ and $\mu_{o}^{2}$ terms in the $\hat{m}_{2}^{2}$ are very different and, since $\hat{m}_{2}^{2} \approx 0$, to obtain radiatively induced gauge symmetry breaking we need $\mu_{o}^{2} \gg M_{o}^{2}$ (as seen in Fig. 5). Hence, the terms $M_{o}^{2}$ and $\mu_{o}^{2}$ in the $\hat{m}_{1}^{2}$ cannot cancel each other and we again get the effective lower limit on $M_{A}$ higher then the present experimental lower limit. Also note that similar arguments explain the correlation between light neutralinos, with large gaugino content, and heavy pseudoscalars which is observed for $M_{t}=180 \mathrm{GeV}$.

For even larger values of $\tan \beta$ the situation again looks differently: as will be discussed in more detail in the next section, for $\tan \beta \approx m_{t} / m_{b}$ all the coefficients in the expression for $\hat{m}_{1}^{2}$ are very close to the coefficients for $\hat{m}_{2}^{2}$ and a light pseudoscalar $A$ becomes compatible both with radiative symmetry breaking and with the experimental constraints.

In general, we recall that the relative magnitude of the coefficients of the $M_{o}^{2}$ and

$\mu_{o}^{2}$ terms in the expressions for $\hat{m}_{1}^{2}$ and $\hat{m}_{2}^{2}$ is responsible for most of the structure which follows from the mechanism of the symmetry breaking alone. The lower limit on $M_{A}$ is an example of its interplay with experimental constraints, for changing values of $M_{t}$ and $\tan \beta$.

In Table 2 we present several examples of the generic sparticle spectra obtained in this model. In Table 3 we also give results obtained with and without inclusion of radiative corrections for the same sets of the input parameters. We see that the difference between the two sets can be very important.

\section{Solutions for Large $\tan \beta$}

It has been discussed for some time ${ }^{[21]}$ that the observed pattern of the quark masses, namely strong isotopic spin symmetry breaking in the third generation electroweak doublet, can be understood as the property of the vacuum. In models with two (or more Higgs doublets) such that two different vevs $v_{1}$ and $v_{2}$ are driving 
the down - and up - quark masses, it is conceivable that $\tan \beta \equiv v_{2} / v_{1} \cong m_{t} / m_{b}$, with the top and bottom Yukawa couplings (almost) equal. Such a scenario is particularly interesting in the context of supersymmetric grand unified models (they require at least two Higgs doublets in the low energy effective lagrangian) which, if based on the groups $S O(10)$ or $E_{6}$ with simple Higgs structure, predict $Y_{t}=Y_{b}$ at the unification scale. This scenario has recently been strongly advocated in a detailed study of the fermion mass and mixing textures ${ }^{[22]}$.

An interesting question is whether the minimum of the potential with large $\tan \beta$ can be obtained by radiative gauge symmetry breaking. The answer to this question is indeed positive, as shown for the first time in ref. [16] and subsequently investigated in a number of papers ${ }^{[19]}$.

Our bottom to top approach is particularly suitable for a systematic search for large $\tan \beta$ solutions and a study of their low energy properties. A sample of such solutions is shown in Fig. $14-17$ for $M_{t}=130 \mathrm{GeV}, \tan \beta=30$ and 31 , and $M_{t}=180 \mathrm{GeV}, \quad \tan \beta=50$ and 51 . The most important points about the large $\tan \beta$ solutions are the following: The degree of fine tuning is rapidly increasing when we approach the value of $\tan \beta$ such that $Y_{t}^{o}=Y_{b}^{o}$. For instance, for $M_{t}=130 \mathrm{GeV}$ and $\tan \beta=31$ (the corresponding value of the $Y_{t}^{o} / Y_{b}^{o}$ at $M_{X}$ is 1.04) we have not found any solutions with $\max \left|\Delta_{i j}\right|<100$ whereas for tan $=30\left(Y_{t}^{o} / Y_{b}^{o} \approx 1.08\right)$ there exist solutions with $\max \left|\Delta_{i j}\right|<30$. This is easily understandable from inspection of the RG equations: the masses $m_{1}^{2}$ and $m_{2}^{2}$ run now very similarly and in consequence the coefficients for $\hat{m}_{1}^{2}$ and $\hat{m}_{2}^{2}$ in eqs. (2.15) are very close to each other. As an example, for $M_{t}=130 \mathrm{GeV}, \quad \tan \beta=31$ and $M_{S U S Y} \cong 460 \mathrm{GeV}$ we have:

$$
\begin{aligned}
& \hat{m}_{1}^{2}=-0.95 M_{o}^{2}-0.27 A_{o}^{2}+0.41 m_{o}^{2}+1.20 \mu^{2}+0.47 M_{o} A_{o} \\
& \hat{m}_{2}^{2}=-1.07 M_{o}^{2}-0.10 A_{o}^{2}+0.45 m_{o}^{2}+1.20 \mu^{2}+0.39 M_{o} A_{o} \\
& \hat{m}_{3}^{2}=0.18 \mu M_{o}-0.41 \mu_{o} A_{o}+1.08 \mu_{o} B_{o}
\end{aligned}
$$

At the same time, the input values of $\hat{m}_{i}^{2}$ 's at $M_{Z}$ are: $\hat{m}_{1}^{2} \approx(6-9) \times 10^{3} \mathrm{GeV}^{2}, \hat{m}_{2}^{2} \approx$ $-5 \times 10^{3} \mathrm{GeV}^{2}$ and $\hat{m}_{3}^{2} \approx 70-160 \mathrm{GeV}^{2}$. Clearly, we need large values of the parameters at $M_{X}$ (in particular of $\left.M_{o}\right) \boxplus$ and a sizable amount of fine tuning to solve those equations.

The above example illustrates several other points typical for the large $\tan \beta$ scenario. These are: $\hat{m}_{3}^{2} \approx 0$ (see eq. (A.5) and small pseudoscalar mass $M_{A}^{2} \approx$ $\hat{m}_{1}^{2}+\hat{m}_{2}^{2}$. The acceptable solutions exist now for small values of $M_{A}$, since for large $\tan \beta$ all the coefficients in the equations for $\hat{m}_{1}^{2}$ and $\hat{m}_{2}^{2}$ are close to each other and moreover, in the combination $M_{A}^{2}=\hat{m}_{1}^{2}+\hat{m}_{2}^{2}$ the terms $M_{o}^{2}$ and $\mu_{o}^{2}$ tend to cancel each other. Therefore, the arguments of the previous Section do not apply: large $\tan \beta$ solutions in general give light pseudoscalar Higgs bosons and a much heavier spectrum for squarks and gluinos.

Another point is that $\hat{m}_{3}^{2} \approx 0$ can be realized in two different ways: small $\mu_{o}$ or cancellations between the three terms. It is the latter possibility which is chosen by most of the solutions.

\footnotetext{
${ }^{11}$ The small differences in the coefficients are driven by the residual difference in the Yukawa coupling and also by the different $U(1)$ charge assignement for the right-handed up- and downsquarks. The latter (and the slepton contributions) are sufficient for the existence of solutions even for $Y_{t}^{o}=Y_{b}^{o}$ but at the expense of very large values of $M_{o}$ and $\max \left|\Delta_{i j}\right| \sim \mathrm{O}\left(10^{3}\right)$.
} 
Finally, we stress that large $\tan \beta$ solutions are particularly sensitive to radiative corrections included in our calculation.

In conclusion, large $\tan \beta$ scenario is compatible with radiative gauge symmetry breaking, although the exact equality $Y_{t}^{o}=Y_{b}^{o}$ can be achieved only at the expense of very strong fine tuning. On the other hand, allowing for, say, (5-10)\% difference in the two Yukawa couplings at $M_{X}$ brings the necessary degree of fine tuning into acceptable range. It is quite possible that such difference is generated by high energy thresholds and contributions from higher dimension operators, but their impact on the Yukawa coupling unification has not been investigated so far.

\section{Conclusions}

In this paper we have proposed a bottom-up approach to unified supergravity models which give the minimal supersymmetric standard model as the effective low energy theory. In this approach the supergravity models are parametrized in terms of a number of low energy physical parameters. The minimal model, with universal boundary conditions at the unification scale and five free mass parameters, has been studied in detail. With more than five low energy input parameters, our set of equations can be used to investigate non-minimal models, which do not assume the universal boundary conditions at $M_{X}$. This will be particularly interesting once some sparticles are discovered and their masses known.

Even in the minimal model, there are several virtues of the bottom-up approach. One is that the number of free parameters is reduced to four (plus the top quark mass) since the mass $M_{Z}$ is known. Secondly, a systematic and complete investigation of the low energy predictions of the model can be easily performed, by scanning the space of four unknown low energy input parameters. Finally, with a suitable choice of the input parameters, our approach is most convenient for inclusion of radiative corrections generated by large Yukawa couplings and particle-sparticle mass splitting: by choosing the soft stop masses as the input parameters the dominant 1-loop radiative corrections are precisely known directly in terms of the input parameters.

The complete inclusion of radiative corrections generated by large Yukawa couplings and particle-sparticle mass splitting is the other main point of this work. They are found to have strong effects on the degree of fine tuning needed in the model and on the final results.

In the above framework, low energy phenomenology of the minimal model has been explored in some detail. In particular, we have imposed all the presently available experimental constraints on the predictions of the model and obtained complete information on those regions of the low energy physical observables which are interesting for future experimental exploration.

Due to the very rigid structure of the minimal model, both the requirement of radiative gauge symmetry breaking and the experimental constraints have strong "global" effects: they introduce a lot of correlations between different quantities predicted by the model, as discussed in the previous sections. Particularly interesting and illuminating is the dependence of the predictions on the values of the top quark 
mass and $\tan \beta$. In this respect, our result based on numerical solutions to the RG equations show a very rich structure, which is difficult to see fully in appproximate analytic calculations. On the list of interesting results are, among others, neutralino as the lightest neutral sparticle, the gaugino content of the lightest neutralino, the lower limit for the pseudoscalar mass $M_{A}$ and, in general, correlations among masses (and couplings) of sparticles. The model predicts a rich sparticle spectrum to be accesible at the next generation of colliders.

In the subsequent paper ${ }^{[23]}$ we calculate in the same approach the relic abundance of the lightest neutralino and impose the additional constraint $\Omega h^{2}<1$ on the predictions of the model.

As a further extension of the present work, it will also be very interesting to explore systematically other constraints which may follow from more specific assumptions about physics at the grand unification scale, such as e.g. proton life time constraints in the supersymmetric $S U(5)$ model $^{[24]}$.

\section{ACKNOWLEDGMENTS}

It is a pleasure to acknowledge useful discussions with I. Antoniadis, C. Bachas, P. Binetruy, H.P. Nilles, C. Savoy and S. Raby. 


\section{APPENDIX A}

The minimization of the potential (2.10) gives

$$
\begin{gathered}
\tan ^{2} \beta\left(\hat{m}_{2}^{2}+\lambda_{2} v^{2}\right)=\hat{m}_{1}^{2}+\lambda_{2} v^{2}+\left(\lambda_{1}-\lambda_{2}\right) v_{1}^{2} \\
2 \hat{m}_{3}^{2}=\left[\hat{m}_{1}^{2}+\hat{m}_{2}^{2}+\lambda_{1} v_{1}^{2}+\lambda_{2} v_{2}^{2}+\left(\lambda_{3}+\lambda_{4}\right) v^{2}\right] \sin 2 \beta
\end{gathered}
$$

where $\tan \beta=v_{2} / v_{1}$ and $v^{2}=v_{1}^{2}+v_{2}^{2}$.

The pseudoscalar mass $M_{A}$ reads:

$$
\begin{aligned}
M_{A}^{2} & =\hat{m}_{1}^{2}+\hat{m}_{2}^{2}+\lambda_{1} v_{1}^{2}+\lambda_{2} v_{2}^{2}+\left(\lambda_{3}+\lambda_{4}\right) v^{2} \\
& \approx \hat{m}_{1}^{2}+\hat{m}_{2}^{2}+\Delta \lambda_{2} v_{2}^{2}
\end{aligned}
$$

The last relation holds in the approximation in which we neglect all but the dominant radiative correction, from the top-stop mass splitting, to $\lambda_{2}$. For $\hat{m}_{i}^{2}$ 's we get the following relations in terms of the input parameters $M_{A}, \tan \beta$ and $v^{2}$ :

$$
\begin{aligned}
\hat{m}_{1}^{2} & =M_{A}^{2} \sin ^{2} \beta+\lambda_{1} v^{2} \frac{\tan ^{2} \beta-1}{\tan ^{2} \beta+1}-\left(\lambda_{1}+\lambda_{3}+\lambda_{4}\right) v_{2}^{2} \\
& \approx M_{A}^{2} \sin ^{2} \beta+\frac{1}{2} M_{Z}^{2} \frac{\tan ^{2} \beta-1}{\tan ^{2} \beta+1} \\
\hat{m}_{2}^{2} & =M_{A}^{2} \cos ^{2} \beta-\lambda_{2} v^{2} \frac{\tan ^{2} \beta}{1+\tan ^{2} \beta}-\cos ^{2} \beta\left(\lambda_{3}+\lambda_{4}\right) v^{2} \\
& \approx M_{A}^{2} \cos ^{2} \beta-\frac{1}{2} M_{Z}^{2} \frac{\tan ^{2} \beta-1}{\tan ^{2} \beta+1}-\Delta \lambda_{2} v^{2} \frac{\tan ^{2} \beta}{1+\tan ^{2} \beta} \\
\hat{m}_{3}^{2} & =\frac{1}{2} M_{A}^{2} \sin 2 \beta
\end{aligned}
$$

1-loop corrections from the squark loops to the parameters of the potential (2.10) (calculated from Feynman diagrams in the "symmetric" phase of the model ${ }^{[6]}$ ) are as follows:

$$
\hat{m}_{i}^{2}(e f f)=\hat{m}_{i}^{2}+\Delta m_{i}^{2}, \quad i=1,2,3
$$

where

$$
\begin{aligned}
(4 \pi)^{2} \Delta m_{1}^{2} & =3 \sum_{A}\left[\frac{1}{6} g_{1}^{2} d_{A}+Y_{d}^{A^{2}}\left(a_{o}\left(m_{Q_{A}}^{2}\right)+a_{o}\left(m_{D_{A}}^{2}\right)\right)\right. \\
& \left.+\mu^{2} Y_{u}^{A^{2}} f\left(m_{Q_{A}}^{2}, m_{U_{A}}^{2}\right)+A_{D_{A}}^{2} Y_{d}^{A^{2}} f\left(m_{Q_{A}}^{2}, m_{D_{A}}^{2}\right)\right] \\
(4 \pi)^{2} \Delta m_{2}^{2}= & 3 \sum_{A}\left[-\frac{1}{6} g_{1}^{2} d_{A}+Y_{u}^{A^{2}}\left(a_{o}\left(m_{Q_{A}}^{2}\right)+a_{o}\left(m_{U_{A}}^{2}\right)\right)\right.
\end{aligned}
$$




$$
\begin{aligned}
& \left.+\mu^{2} Y_{d}^{A^{2}} f\left(m_{Q_{A}}^{2}, m_{D_{A}}^{2}\right)+A_{U_{A}}^{2} Y_{u}^{A^{2}} f\left(m_{Q_{A}}^{2}, m_{U_{A}}^{2}\right)\right] \\
(4 \pi)^{2} \Delta m_{3}^{2} & =\frac{3}{2} \mu \sum_{A}\left(A_{U_{A}} Y_{u}^{A^{2}} f\left(m_{Q_{A}}^{2}, m_{U_{A}}^{2}\right)\right. \\
& \left.+A_{D_{A}} Y_{d}^{A^{2}} f\left(m_{Q_{A}}^{2}, m_{D_{A}}^{2}\right)\right)
\end{aligned}
$$

where

$$
\begin{gathered}
f\left(m_{1}^{2}, m_{2}^{2}\right)=-1+\frac{m_{1}^{2}}{m_{1}^{2}-m_{2}^{2}} \log \frac{m_{1}^{2}}{\Lambda^{2}}+\frac{m_{2}^{2}}{m_{2}^{2}-m_{1}^{2}} \log \frac{m_{2}^{2}}{\Lambda^{2}} \\
d_{A} \equiv 2 a_{o}\left(m_{U_{A}}^{2}\right)-a_{o}\left(m_{D_{A}}^{2}\right)-a_{o}\left(m_{Q_{A}}^{2}\right)
\end{gathered}
$$

and

$$
\begin{gathered}
a_{o}\left(m^{2}\right)=(4 \pi)^{2} \int \frac{d^{d} k}{(2 \pi)^{d}} \frac{i}{k^{2}-m^{2}}=m^{2}\left(\eta+\log \frac{m^{2}}{\Lambda^{2}}-1\right) \\
\eta=\frac{2}{d-4}+\gamma_{E}-\log 4 \pi
\end{gathered}
$$

We recall the notation: $\hat{m}_{i}=m_{i}^{2}+\mu^{2}, \quad i=1,2$, where $m_{i}^{2}$ 's are the soft supersymmetry breaking Higgs boson masses and $\mu$ is the supersymmetric Higgs mixing parameter; $\hat{m}_{3}^{2}=B \mu ; Q_{A}$ are left- handed squark doublets (the index $A$ numbers the generations), $U_{A}$ and $D_{A}$ are the up and down right-handed squark $S U(2)$ singlets, respectively; the trilinear soft supersymmetry breaking scalar couplings are: $y_{L}^{A}=Y_{l}^{A} A_{L_{A}}, \quad y_{D}^{A}=Y_{d}^{A} A_{D_{A}}$ and $y_{U}^{A}=Y_{u}^{A} A_{U_{A}}$ where $Y^{\prime}$ 's denote respective Yukawa couplings; $g_{i}$ 's are gauge couplings.

The scale $\Lambda \equiv M_{S U S Y}$ has been chosen so that the term (A.8a) vanishes (see the text).

1-loop corrections to the quartic Higgs couplings are as follows: 


$$
\begin{aligned}
4 \lambda_{1} & =g_{1}^{2}+g_{2}^{2}+\frac{1}{2(4 \pi)^{2}} \sum_{A}\left[\frac{8}{3} g_{1}^{4} \log \frac{m_{U_{A}}^{2}}{\Lambda^{2}}\right. \\
& +\left(\frac{2}{3} g_{1}^{4}-8 g_{1}^{2} Y_{d}^{A^{2}}+24 Y_{d}^{A^{4}}\right) \log \frac{m_{D_{A}}^{2}}{\Lambda^{2}} \\
& \left.+\left(3 g_{2}^{4}+\frac{1}{3} g_{1}^{4}+24 Y_{d}^{A^{4}}-12 g_{2}^{2} Y_{d}^{A^{2}}-4 g_{1}^{2} Y_{d}^{A^{2}}\right) \log \frac{m_{Q_{A}}^{2}}{\Lambda^{2}}\right]
\end{aligned}
$$

$$
\begin{aligned}
4 \lambda_{2} & =g_{1}^{2}+g_{2}^{2}+\frac{1}{2(4 \pi)^{2}} \sum_{A}\left[\frac{2}{3} g_{1}^{4} \log \frac{m_{D_{A}}^{2}}{\Lambda^{2}}\right. \\
& +\left(\frac{8}{3} g_{1}^{4}-16 g_{1}^{2} Y_{u}^{A^{2}}+24 Y_{u}^{A^{4}}\right) \log \frac{m_{U_{A}}^{2}}{\Lambda^{2}} \\
& \left.+\left(3 g_{2}^{4}+\frac{1}{3} g_{1}^{4}+24 Y_{u}^{A^{4}}-12 g_{2}^{2} Y_{u}^{A^{2}}-4 g_{1}^{2} Y_{u}^{A^{2}}\right) \log \frac{m_{Q_{A}}^{2}}{\Lambda^{2}}\right]
\end{aligned}
$$

$$
\begin{aligned}
4 \lambda_{3} & =g_{2}^{2}-g_{1}^{2}+\frac{1}{(4 \pi)^{2}} \sum_{A}\left[12 Y_{u}^{A^{2}} Y_{d}^{A^{2}}\left(f\left(m_{D_{A}}^{2}, m_{U_{A}}^{2}\right)+\log \frac{m_{Q_{A}}^{2}}{\Lambda^{2}}\right)\right. \\
& -g_{1}^{2}\left(\left(Y_{u}^{A^{2}}-Y_{d}^{A^{2}}\right) \log \frac{m_{Q_{A}}^{2}}{\Lambda^{2}}-2 Y_{d}^{A^{2}} \log \frac{m_{D_{A}}^{2}}{\Lambda^{2}}-4 Y_{u}^{A^{2}} \log \frac{m_{U_{A}}^{2}}{\Lambda^{2}}\right) \\
& -\frac{1}{6} g_{1}^{4}\left(\log \frac{m_{Q_{A}}^{2}}{\Lambda^{2}}+2 \log \frac{m_{D_{A}}^{2}}{\Lambda^{2}}+8 \log \frac{m_{U_{A}}^{2}}{\Lambda^{2}}\right) \\
& \left.+\left(\frac{3}{2} g_{2}^{4}-3 g_{2}^{2}\left(Y_{u}^{A^{2}}+Y_{d}^{A^{2}}\right)\right) \log \frac{m_{Q_{A}}^{2}}{\Lambda^{2}}\right]
\end{aligned}
$$

$$
\begin{aligned}
2 \lambda_{4} & =-g_{2}^{2}-\frac{1}{(4 \pi)^{2}} \sum_{A}\left[\left(\frac{3}{2} g_{2}^{4}-3 g_{2}^{2}\left(Y_{u}^{A^{2}}+Y_{d}^{A^{2}}\right)\right) \log \frac{m_{Q_{A}}^{2}}{\Lambda^{2}}\right. \\
& \left.+6 Y_{u}^{A^{2}} Y_{d}^{A^{2}}\left(\log \frac{m_{Q_{A}}^{2}}{\Lambda^{2}}+f\left(m_{D_{A}}^{2}, m_{U_{A}}^{2}\right)\right)\right]
\end{aligned}
$$




\section{APPENDIX B}

For easy reference we list here the 1-loop supersymmetric renormalization group equations for the Yukawa couplings and for the soft supersymmetry breaking parameters $^{[19]}$.

$$
\begin{aligned}
& 2 \frac{d}{d t} Y_{l}^{A}=Y_{l}^{A}\left(3 g_{1}^{2}+3 g_{2}^{2}-3 Y_{l}^{A^{2}}-\sum_{B}\left(Y_{l}^{B^{2}}+3 Y_{d}^{B^{2}}\right)\right) \\
& 2 \frac{d}{d t} Y_{d}^{A}=Y_{d}^{A}\left(\frac{16}{3} g_{3}^{2}+\frac{7}{9} g_{1}^{2}+3 g_{2}^{2}-3 Y_{d}^{A^{2}}-Y_{u}^{A^{2}}-\sum_{B}\left(Y_{l}^{B^{2}}+3 Y_{d}^{B^{2}}\right)\right) \\
& 2 \frac{d}{d t} Y_{u}^{A}=Y_{u}^{A}\left(\frac{16}{3} g_{3}^{2}+\frac{13}{9} g_{1}^{2}+3 g_{2}^{2}-3 Y_{u}^{A^{2}}-Y_{d}^{A^{2}}-3 \sum_{B} Y_{u}^{B^{2}}\right) \\
& \frac{d}{d t} m_{L_{A}}^{2}=3 g_{2}^{2} M_{2}^{2}+g_{1}^{2} M_{1}^{2}-Y_{l}^{A^{2}}\left(m_{L_{A}}^{2}+m_{E_{A}}^{2}+m_{1}^{2}+A_{L_{A}}^{2}\right) \\
& \frac{d}{d t} m_{E_{A}}^{2}=4 g_{1}^{2} M_{1}^{2}-2 Y_{l}^{A^{2}}\left(m_{L_{A}}^{2}+m_{E_{A}}^{2}+m_{1}^{2}+A_{L_{A}}^{2}\right) \\
& \frac{d}{d t} m_{Q_{A}}^{2}=\frac{16}{3} g_{3}^{2} M_{3}^{2}+3 g_{2}^{2} M_{2}^{2}+\frac{1}{9} g_{1}^{2} M_{1}^{2}-Y_{d}^{A^{2}}\left(m_{Q_{A}}^{2}+m_{D_{A}}^{2}+m_{1}^{2}+A_{D_{A}}^{2}\right) \\
& -Y_{u}^{A^{2}}\left(m_{Q_{A}}^{2}+m_{U_{A}}^{2}+m_{2}^{2}+A_{U_{A}}^{2}\right) \\
& \frac{d}{d t} m_{D_{A}}^{2}=\frac{16}{3} g_{3}^{2} M_{3}^{2}+\frac{4}{9} g_{1}^{2} M_{1}^{2}-2 Y_{d}^{A^{2}}\left(m_{Q_{A}}^{2}+m_{D_{A}}^{2}+m_{1}^{2}+A_{D_{A}}^{2}\right) \\
& \frac{d}{d t} m_{U_{A}}^{2}=\frac{16}{3} g_{3}^{2} M_{3}^{2}+\frac{16}{9} g_{1}^{2} M_{1}^{2}-2 Y_{u}^{A^{2}}\left(m_{Q_{A}}^{2}+m_{U_{A}}^{2}+m_{2}^{2}+A_{U_{A}}^{2}\right) \\
& \frac{d}{d t} m_{1}^{2}=3 g_{2}^{2} M_{2}^{2}+g_{1}^{2} M_{1}^{2}-3 \sum_{A} Y_{d}^{A^{2}}\left(m_{Q_{A}}^{2}+m_{D_{A}}^{2}+m_{1}^{2}+A_{D_{A}}^{2}\right) \\
& -\sum_{A} Y_{l}^{A^{2}}\left(m_{L_{A}}^{2}+m_{E_{A}}^{2}+m_{1}^{2}+A_{L_{A}}^{2}\right) \\
& \frac{d}{d t} m_{2}^{2}=3 g_{2}^{2} M_{2}^{2}+g_{1}^{2} M_{1}^{2}-3 \sum_{A} Y_{u}^{A^{2}}\left(m_{Q_{A}}^{2}+m_{U_{A}}^{2}+m_{2}^{2}+A_{U_{A}}^{2}\right) \\
& \frac{d}{d t} \mu=\frac{1}{2} \mu\left(3 g_{2}^{2}+g_{1}^{2}-\sum_{B}\left(Y_{l}^{B^{2}}+3 Y_{d}^{B^{2}}+3 Y_{u}^{B^{2}}\right)\right)
\end{aligned}
$$




$$
\begin{aligned}
& \frac{d}{d t} B=-\left(3 g_{2}^{2} M_{2}+g_{1}^{2} M_{1}\right)-\sum_{B}\left(A_{L_{B}} Y_{l}^{B^{2}}+3 A_{D_{B}} Y_{d}^{B^{2}}+3 A_{U_{B}}^{2} Y_{u}^{B^{2}}\right) \\
& \frac{d}{d t} A_{L_{A}}=-\left(3 g_{2}^{2} M_{2}+g_{1}^{2} M_{1}\right)-3 A_{L_{A}}^{2} Y_{l}^{A^{2}}-\sum_{B}\left(A_{L_{B}}^{2} Y_{l}^{B^{2}}+3 A_{D_{B}} Y_{d}^{B^{2}}\right) \\
& \frac{d}{d t} A_{D_{A}}=-\left(\frac{16}{3} g_{3}^{2} M_{3}+3 g_{2}^{2} M_{2}+\frac{7}{9} g_{1}^{2} M_{1}\right)-A_{U_{A}} Y_{u}^{A^{2}}-3 A_{D_{A}} Y_{d}^{A^{2}} \\
& -\sum_{B}\left(A_{L_{B}} Y_{l}^{B^{2}}+3 A_{D_{B}} Y_{d}^{B^{2}}\right) \\
& \frac{d}{d t} A_{U_{A}}=-\left(\frac{16}{3} g_{3}^{2} M_{3}+3 g_{2}^{2} M_{2}+\frac{13}{9} g_{1}^{2} M_{1}\right)-3 A_{U_{A}} Y_{u}^{A^{2}}-A_{D_{A}} Y_{d}^{A^{2}} \\
& -3 \sum_{B} A_{U_{B}} Y_{u}^{B^{2}} \\
& \frac{d}{d t}\left(\frac{M_{i}}{g_{i}^{2}}\right)=0 \\
& \frac{d}{d t} g_{i}=b_{i} g_{i}^{3}
\end{aligned}
$$

Here $t=\frac{1}{(4 \pi)^{2}} \ln \frac{M_{X}^{2}}{Q^{2}}$ and $b_{1}=1+10 n_{G} / 3, \quad b_{2}=5-2 n_{G}, b_{3}=-9+2 n_{G}$. The above equations are used in the range $\left(M_{X}, M_{S U S Y}\right)$. In the range $\left(M_{S U S Y}, M_{Z}\right)$ (i.e. after decoupling of squarks and gluinos we use the equations given in ref. [6]. 


\section{REFERENCES}

[1] L.E. Ibánez and G.G. Ross, Phys. Lett. B110, 215 (1982);

K. Inoue et al., Prog. Theor. Phys. 68, 927 (1982);

L. Alvarez-Gaumé, J. Polchinsky and M. Wise, Nucl. Phys. B221, 495 (1983);

J. Ellis, J. Hagelin, D. Nanopoulos and K. Tamvakis, Phys. Lett. B125, 275 (1983);

L.E. Ibánez and G. López, Nucl. Phys. B233, 511 (1984).

[2] R. Barbieri and G.F. Giudice, Nucl. Phys. B306, 63 (1988);

G.G. Ross and R.G. Roberts, Nucl. Phys. B377, 571 (1992).

[3] M. Dress and M.M. Nojiri, DESY preprint, Desy 92-101, Slac Pub-5860, July 1992.

[4] G. Gamberini, G. Ridolfi and F. Zwirner, Nucl. Phys. B331, 331 (1990);

P.H. Chankowski, Phys. Rev. D41, 2877 (1990).

[5] Y. Okada, M. Yamaguchi and T. Yanagida, Prog. Theor. Phys. 85, 1 (1991);

H.E. Haber and R. Hempfling, Phys. Rev. Lett. 66, 1815 (1991);

J. Ellis, G. Ridolfi and F. Zwirner, Phys. Lett. B257, 83 (1991);

R. Barbieri, M. Frigeni and F. Caravaglio, Phys. Lett. B258, 395 (1991).

[6] P.H. Chankowski. Phys. Rev. D41, 2877 (1990).

[7] M.A. Diaz and H.E. Haber, Phys. Rev. D45, 4246 (1991);

K. Sasaki, M. Carena and C.E.M. Wagner, Nucl. Phys. B381, 66 (1992);

P.H. Chankowski, S. Pokorski and J. Rosiek, Phys. Lett. B281, 100 (1992);

H.E. Haber and R. Hempfling, Santa Cruz preprint SCIPP-91/33.

[8] J. Ellis, S. Kelly and D.V. Nanopoulos, Phys. Lett. 260B, 131 (1991);

U. Amaldi, W. deBoer and M. Furstenau, Phys. Lett. 260B, 447 (1991);

P. Langacker and M. Luo, Phys. Rev. D44, 817 (1991);

F. Anselmo, L. Cifarelli, A. Peterman and A. Zichichi, Nuovo Cimento 104A, 1817 (1991);

G.G. Ross and R.G. Roberts, Nucl. Phys. B377, 571 (1992).

[9] P. Langacker and N. Polonsky, University of Pennsylvania preprint, UPR0513T.

[10] M. Carena, S. Pokorski and C.E.M. Wagner, Max-Planck-Institute preprint, MPI-Ph/93-10.

[11] H. Arason, D.J. Castano, B. Keszthelyi, S. Mikaelian, E.J. Piard, P. Ramond and B.D. Wright, University of Florida preprint UFIFT-HEP-91-33.

[12] J.R. Espinosa and M. Quirós, Phys. Lett. B266, 389 (1991). 
[13] M. Carena, T.E. Clark, C.E.M. Wagner, W.A. Bardeen, K. Sasaki, Nucl. Phys. B369, 33 (1992).

[14] A. Bouquet, J. Kaplan and C.A. Savoy, Nucl. Phys. B262, 299 (1985).

[15] J.M. Frère, D.R.T. Jones and S. Raby, Nucl. Phys. B222, 11 (1983);

M. Claudson, L. Hall and I. Hinchliffe, Nucl. Phys. B228, 501 (1983);

J.P. Derendinger and C.A. Savoy, Nucl. Phys. B237, 307 (1984).

[16] M. Olechowski and S. Pokorski, Phys. Lett. B214, 393 (1988);

S. Pokorski, "How is the isotopic spin symmetry of quark masses broken ?" Proceedings of the XIIth International Workshop on Weak Interactions and Neutrinos, Ginosar, Israel, April 1989 and "On weak isospin breaking in the quark mass spectrum" in Festschrift for Leon Van Hove, World Scientific, Singapore, 1990.

[17] See e.g. K. Griest, M. Kamionkowski and M. Turner, Phys. Rev. D41, 3565 (1990).

[18] M. Drees and K. Hikasa, Phys. Lett. B252, 127 (1990).

[19] H.P. Nilles, "Beyond the Standard Model", in Proceedings of the 1990 Theoretical Advanced Study Institute in Elementary Particle Physics, p. 633; Eds. M. Cvetic and P. Langacker, World Scientific; P.H. Chankowski, Diploma Thesis (1990), University of Warsaw;

A. Seidl, Diploma Thesis (1990), Technical University, Munich;

W. Majerotto and B. Mösslacher, Z. Phys. C48, 273 (1990);

S. Bertolini, F. Borzumati, A. Masiero and G. Ridolfi, Nucl. Phys. B353, 591 (1991);

M. Drees and M.M. Nojiri, Nucl. Phys. B369, 54 (1992);

B. Ananthanarayan, G. Lazarides and Q. Shafi, Bartol Research Institute preprint BA-92-29.

[20] K. Inoue, A. Kakuto, H. Komatsu and S. Takeshita, Prog. Theor. Phys. 67, 889 (1982) and 68, 927 (1983);

B. Gato, J. Leon, J. Perez-Mercader and M. Quiros, Nucl. Phys. B253, 285 (1985);

N.K. Falck, Z. Phys. C30, 247 (1986).

[21] See for instance, G,C. Branco, A.J. Buras and J.M. Gerard, Nucl. Phys. B259, 306 (1985);

P. Krawczyk and S. Pokorski, Phys. Rev. Lett. 60, 182 (1988);

S. Pokorski, "How is the isotopic spin symmetry of quark masses broken ?" Proceedings of the XIIth Intern. Workshop on Weak Interactions and Neutrinos, Ginosar, Israel, April 1989.

[22] S. Dimopoulos, L.J. Hall and S. Raby, Phys. Rev. Lett. 68. 1984 (1992); Phys. Rev. D45, 4192 (1992);

G.W. Anderson, S. Raby, S. Dimopoulos and L. Hall, preprint OHSTPYHEP-92-018, October 1992. 
[23] P. Gondolo, M. Olechowski and S. Pokorski, "Neutralinos as dark matter in supergravity models", preprint MPI-Ph/92-81, September 1992, to appear in Proceedings of the XXVI International Conference on High Energy Physics, Dallas, August 1992;

Max-Planck-Institute for Physics preprint, to be published.

[24] R. Arnowitt and P. Nath, Phys. Rev. Lett. 69, 725 (1992);

P. Nath and R. Arnowitt, Phys. Lett. B289, 368 (1992). 


\section{TABLE 1}

Coefficients in Eqs. (2.15-2.17) for $M_{S U S Y}=300 \mathrm{GeV}$.

The numbers in brackets are without radiative corrections $\left(M_{S U S Y}=M_{Z}\right)$.

\begin{tabular}{|c|c|c|c|c|c|c|}
\hline \multicolumn{2}{|c|}{$\hat{m}_{1}^{2}$} & $M_{o}^{2}$ & $A_{o}^{2}$ & $m_{o}^{2}$ & $\mu_{o}^{2}$ & $M_{o} A_{o}$ \\
\hline \multirow{6}{*}{$M_{t}=130$} & \multirow[t]{2}{*}{$\tan \beta=2$} & .478 & -.002 & .996 & 1.461 & .005 \\
\hline & & $(.478)$ & $(-001)$ & $(.997)$ & $(1.407)$ & $(.004)$ \\
\hline & \multirow[t]{2}{*}{$\tan \beta=10$} & .286 & -.041 & .931 & 1.541 & .092 \\
\hline & & $(.222)$ & $(-.022)$ & $(.928)$ & $(1.495)$ & $(.087)$ \\
\hline & \multirow[t]{2}{*}{$\tan \beta=30$} & -.961 & -.223 & .438 & 1.220 & .441 \\
\hline & & $(-1.401)$ & $(-.116)$ & $(.414)$ & $(1.184)$ & $(.452)$ \\
\hline \multirow{6}{*}{$\underline{M_{t}=180}$} & \multirow[t]{2}{*}{$\tan \beta=2$} & .480 & -.002 & .996 & .444 & .004 \\
\hline & & $(.482)$ & $(-.001)$ & $(.997)$ & $(.411)$ & $(.003)$ \\
\hline & \multirow[t]{2}{*}{$\tan \beta=10$} & .316 & -.038 & .939 & .963 & .079 \\
\hline & & $(.267)$ & $(-.018)$ & $(.937)$ & $(.906)$ & $(.070)$ \\
\hline & \multirow[t]{2}{*}{$\tan \beta=30$} & -.765 & -.215 & .503 & .761 & .400 \\
\hline & & $(-1.128)$ & $(-.101)$ & $(.484)$ & $(.717)$ & $(.381)$ \\
\hline \multicolumn{2}{|c|}{$\hat{m}_{2}^{2}$} & $\overline{M_{o}^{2}}$ & $\overline{A_{o}^{2}}$ & $m_{o}^{2}$ & $\mu_{o}^{2}$ & $M_{o} A_{o}$ \\
\hline \multirow{6}{*}{$\underline{M_{t}}=130$} & \multirow[t]{2}{*}{ 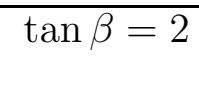 } & -1.580 & -.118 & .296 & 1.406 & .478 \\
\hline & & $(-2.176)$ & $(-.125)$ & $(.260)$ & $(1.407)$ & $(.574)$ \\
\hline & \multirow[t]{2}{*}{$\tan \beta=10$} & -1.235 & -.110 & .434 & 1.499 & .445 \\
\hline & & $(-1.756)$ & $(-.118)$ & $(.403)$ & $(1.495)$ & $(.545)$ \\
\hline & \multirow[t]{2}{*}{$\tan \beta=30$} & -1.198 & -.102 & .436 & 1.218 & .413 \\
\hline & & $(-1.688)$ & $(-.108)$ & $(.408)$ & $(1.184)$ & $(.499)$ \\
\hline \multirow{6}{*}{$\underline{M_{t}}=180$} & \multirow[t]{2}{*}{$\tan \beta=2$} & -2.767 & -.022 & -.401 & .409 & .088 \\
\hline & & $(-3.523)$ & $(-.021)$ & $(-.435)$ & $(.411)$ & $(.095)$ \\
\hline & \multirow[t]{2}{*}{$\tan \beta=10$} & -2.406 & -.085 & -.129 & .906 & .343 \\
\hline & & $(-3.121)$ & $(-.084)$ & $(-.167)$ & $(.906)$ & $(.386)$ \\
\hline & \multirow[t]{2}{*}{$\tan \beta=30$} & -2.340 & -.072 & -.123 & .733 & .292 \\
\hline & & $(-3.017)$ & $(-.070)$ & $(-.155)$ & $(.717)$ & $(.322)$ \\
\hline
\end{tabular}




\begin{tabular}{|c|c|c|c|c|c|c|}
\hline \multicolumn{2}{|c|}{$m_{Q}^{2}$} & $M_{o}^{2}$ & 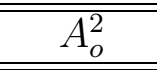 & 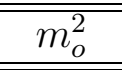 & 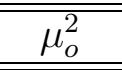 & 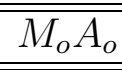 \\
\hline \multirow{5}{*}{$\underline{M_{t}}=130$} & $\tan \beta=2$ & $\begin{array}{c}5.568 \\
(6.358)\end{array}$ & $\begin{array}{c}-.042 \\
(-.042)\end{array}$ & $\begin{array}{l}.767 \\
(.772)\end{array}$ & $\begin{array}{l}.000 \\
(.000)\end{array}$ & $\begin{array}{c}.175 \\
(.193)\end{array}$ \\
\hline & \multirow[t]{2}{*}{$\tan \beta=10$} & 5.623 & -.044 & .796 & .000 & .185 \\
\hline & & (6.413) & $(-.046)$ & $(.780)$ & $(.000)$ & $(.210)$ \\
\hline & \multirow[t]{2}{*}{$\tan \beta=30$} & 5.196 & -.066 & .659 & .000 & .281 \\
\hline & & (5.898) & $(-.068)$ & $(.636)$ & $(.000)$ & $(.313)$ \\
\hline \multirow{6}{*}{$\underline{M_{t}=180}$} & \multirow[t]{2}{*}{$\tan \beta=2$} & 5.142 & -.008 & .524 & .000 & .033 \\
\hline & & (5.910) & $(-.007)$ & $(.521)$ & $(.000)$ & $(.033)$ \\
\hline & \multirow[t]{2}{*}{$\tan \beta=10$} & 5.219 & -.034 & .605 & .000 & . 145 \\
\hline & & $(5.974)$ & $(-.033)$ & $(.593)$ & $(.000)$ & $(.151)$ \\
\hline & \multirow[t]{2}{*}{$\tan \beta=30$} & 4.866 & -.051 & .489 & .000 & .216 \\
\hline & & (5.546) & $(-.050)$ & $(.471)$ & $(.000)$ & $(.231)$ \\
\hline \multicolumn{2}{|c|}{$m_{U}^{2}$} & $M_{o}^{2}$ & $A_{o}^{2}$ & $m_{o}^{2}$ & $\mu_{o}^{2}$ & $M_{o} A_{o}$ \\
\hline \multirow{6}{*}{$\underline{M_{t}}=130$} & \multirow[t]{2}{*}{$\overline{\tan \beta=2}$} & 4.395 & $\begin{array}{l}-.083 \\
\end{array}$ & .536 & .000 & .348 \\
\hline & & $(5.019)$ & $(-.083)$ & $(.507)$ & $(.000)$ & $(.383)$ \\
\hline & \multirow[t]{2}{*}{$\tan \beta=10$} & 4.640 & -.077 & .629 & .000 & .323 \\
\hline & & $(5.299)$ & $(-.079)$ & $(.602)$ & $(.000)$ & $(.363)$ \\
\hline & \multirow[t]{2}{*}{$\tan \beta=30$} & 4.669 & -.071 & .631 & .000 & .299 \\
\hline & & $(5.344)$ & $(-.072)$ & $(.605)$ & $(.000)$ & $(.332)$ \\
\hline \multirow{5}{*}{$\underline{M_{t}}=180$} & $\tan \beta=2$ & $\begin{array}{c}3.535 \\
(4.121)\end{array}$ & $\begin{array}{l}-.015 \\
(-.014)\end{array}$ & $\begin{array}{c}.048 \\
(.043)\end{array}$ & $\begin{array}{c}.000 \\
(.000)\end{array}$ & $\begin{array}{c}.065 \\
(.063)\end{array}$ \\
\hline & \multirow[t]{2}{*}{$\tan \beta=10$} & 3.803 & -.060 & .242 & .000 & .252 \\
\hline & & (4.389) & $(-.056)$ & $(.222)$ & $(.000)$ & $(.258)$ \\
\hline & \multirow[t]{2}{*}{$\tan \beta=30$} & 3.853 & -.051 & .247 & .000 & .213 \\
\hline & & $(4.458)$ & $(-.047)$ & $(.230)$ & $(.000)$ & $(.215)$ \\
\hline
\end{tabular}

\begin{tabular}{|c|c|c|c|c|}
\hline \multicolumn{2}{|c|}{$\hat{m}_{3}^{2}$} & $\mu_{o} M_{o}$ & $\overline{\mu_{o} A_{o}}$ & $\mu_{o} B_{o}$ \\
\hline \multirow{6}{*}{$\underline{M_{t}=130}$} & $\tan \beta=2$ & -.070 & -.273 & 1.186 \\
\hline & & $(.004)$ & $(-.294)$ & (1.186) \\
\hline & $\tan \beta=10$ & -.143 & -.252 & 1.222 \\
\hline & & $(-.073)$ & $(-.273)$ & $(1.223)$ \\
\hline & $\tan \beta=30$ & -.182 & -.408 & 1.088 \\
\hline & & $(-.305)$ & $(-.427)$ & $(1.088)$ \\
\hline \multirow{5}{*}{$M_{t}=180$} & $\tan \beta=2$ & $\begin{array}{c}.290 \\
(.346)\end{array}$ & $\begin{array}{l}-.305 \\
(-.307)\end{array}$ & $\begin{array}{c}.641 \\
(.641)\end{array}$ \\
\hline & $\tan \beta=10$ & .265 & -.378 & .951 \\
\hline & & $(.352)$ & $(-.390)$ & $(.952)$ \\
\hline & $\tan \beta=30$ & .444 & -.463 & .847 \\
\hline & & $(.558)$ & $(-.471)$ & $(.847)$ \\
\hline
\end{tabular}




\section{TABLE 2}

Generic spectra of the supersymmetric particles with the lightest pseudoscalar $A$ or neutralino or chargino (underlined).

\begin{tabular}{|c|c|c|c|c|c|c|}
\hline $\begin{array}{l}M_{t} \\
\tan \beta\end{array}$ & $\begin{array}{r}130 \\
2\end{array}$ & $\begin{array}{r}130 \\
2\end{array}$ & $\begin{array}{r}130 \\
2\end{array}$ & $\begin{array}{r}130 \\
10\end{array}$ & $\begin{array}{r}130 \\
10\end{array}$ & $\begin{array}{r}130 \\
10\end{array}$ \\
\hline 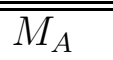 & 230.0 & 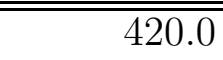 & 380.0 & $\underline{190.0}$ & 300.0 & 2220.0 \\
\hline$m_{Q}$ & 225.0 & 250.0 & 250.0 & 300.0 & 375.0 & 425.0 \\
\hline$m_{U}$ & 185.0 & 150.0 & 170.0 & 260.0 & 315.0 & 385.0 \\
\hline$M_{h}$ & 58.6 & 60.0 & 60.4 & 94.2 & 95.5 & 96.0 \\
\hline$M_{n_{1}}$ & 39.8 & $\underline{32.5}$ & 32.9 & 48.0 & $\underline{45.7}$ & 48.0 \\
\hline$M_{n_{2}}$ & 87.4 & 73.3 & 74.9 & 85.4 & 75.9 & 80.9 \\
\hline$M_{n_{4}}$ & 191.9 & 283.9 & 248.9 & 207.0 & 176.9 & 177.2 \\
\hline$M_{c_{1}}$ & 49.5 & 47.0 & $\underline{45.1}$ & 76.4 & 64.9 & $\underline{60.6}$ \\
\hline$M_{C_{2}}$ & 222.2 & 304.1 & 271.9 & 217.2 & 187.0 & 183.7 \\
\hline$M_{\tilde{t}_{1}}$ & 145.6 & 84.6 & 123.6 & 177.7 & 242.3 & 399.9 \\
\hline$M_{\tilde{t}_{2}}$ & 302.6 & 325.0 & 322.3 & 389.2 & 454.0 & 440.0 \\
\hline$M_{\tilde{q}}$ & $228.6-245.7$ & $253.7-313.5$ & $253.8-302.9$ & $305.5-322.2$ & $379.4-422.8$ & $428.9-443.1$ \\
\hline$M_{\tilde{l}}^{1}$ & $82.8-91.0$ & $247.5-253.0$ & $233.3-239.0$ & $86.7-113.5$ & 283.9-301.2 & $186.4-234.3$ \\
\hline$\overline{M_{t}}$ & 180 & 180 & 180 & 180 & 180 & 180 \\
\hline $\tan \beta$ & 2 & 2 & 2 & 30 & 30 & 30 \\
\hline$M_{A}$ & $\underline{300.0}$ & 550.0 & 355.0 & $\underline{175.0}$ & 535.0 & 185.0 \\
\hline$m_{Q}$ & 225.0 & 300.0 & 225.0 & 300.0 & 525.0 & 325.0 \\
\hline$m_{U}$ & 165.0 & 160.0 & 145.0 & 260.0 & 345.0 & 285.0 \\
\hline$M_{h}$ & 75.6 & 79.9 & 74.5 & 111.7 & 117.7 & 113.0 \\
\hline$M_{n_{1}}$ & 37.7 & $\underline{22.6}$ & 32.9 & 47.4 & $\underline{22.5}$ & 52.1 \\
\hline$M_{n_{2}}$ & 83.5 & 47.6 & 74.7 & 86.6 & 43.8 & 95.1 \\
\hline$M_{n_{4}}$ & 231.0 & 379.6 & 251.0 & 244.3 & 407.6 & 250.6 \\
\hline$M_{C_{1}}$ & 51.6 & 63.7 & $\underline{45.3}$ & 88.5 & $\underline{45.2}$ & 97.1 \\
\hline$M_{C_{2}}$ & 256.1 & 373.5 & 273.7 & 246.5 & 411.2 & 252.4 \\
\hline$M_{\tilde{t}_{1}}$ & 233.1 & 138.1 & 224.4 & 232.1 & 361.6 & 258.9 \\
\hline$M_{\tilde{t}_{2}}$ & 285.5 & 392.5 & 281.6 & 398.7 & 563.9 & 418.2 \\
\hline$M_{\tilde{q}}$ & $229.2-260.4$ & $303.4-386.9$ & $229.4-275.0$ & $284.5-353.6$ & $524.3-809.2$ & $309.9-371.5$ \\
\hline$M_{\tilde{l}}^{1}$ & $137.2-143.4$ & $332.9-339.0$ & $194.1-200.6$ & $89.6-177.4$ & 549.3-798.4 & $\underline{82.5}-174.4$ \\
\hline
\end{tabular}




\section{Table 3}

Comparison of the supersymmetric particle spectra, for the same sets of the input parameters,

with and without (the numbers in brackets) radiative corrections

\begin{tabular}{|l|rrrr|}
\hline \hline$M_{t}$ & 130 & 130 & 180 & 180 \\
$\tan \beta$ & 2 & 10 & 2 & 30 \\
\hline \hline$M_{A}$ & 233.0 & 228.0 & 465.0 & 315.0 \\
$m_{Q}$ & 225.0 & 425.0 & 400.0 & 325.0 \\
$m_{U}$ & 185.0 & 385.0 & 320.0 & 225.0 \\
\hline$M_{h}$ & $58.6(52.0)$ & $96.0(88.9)$ & $90.1(54.0)$ & $111.1(90.8)$ \\
$M_{n_{1}}$ & $38.5(40.4)$ & $56.2(65.1)$ & $60.3(60.2)$ & $32.1(51.8)$ \\
$M_{n_{2}}$ & $85.4(87.9)$ & $89.5(117.5)$ & $115.5(114.1)$ & $61.3(100.3)$ \\
$M_{n_{4}}$ & $184.6(208.1)$ & $186.1(244.6)$ & $396.0(423.6)$ & $292.0(426.0)$ \\
$M_{c_{1}}$ & $46.4(52.7)$ & $74.8(109.0)$ & $134.0(131.2)$ & $59.3(98.8)$ \\
$M_{C_{2}}$ & $215.7(236.1)$ & $193.7(253.6)$ & $385.7(414.6)$ & $298.3(430.3)$ \\
$M_{\tilde{t}}$ & $149.4(143.5)$ & $344.8(333.0)$ & $262.7(241.5)$ & $228.4(159.1)$ \\
$M_{\tilde{t}}$ & $300.8(304.8)$ & $484.3(495.0)$ & $500.4(512.4)$ & $399.1(433.3)$ \\
$M_{\tilde{q}}$ & $228.8-246.7$ & $428.8-441.6$ & $402.5-439.0$ & $323.6-494.0$ \\
& $(228.4-245.1)$ & $(428.9-439.5)$ & $(402.0-438.9)$ & $(312.0-534.4)$ \\
$M_{\tilde{l}}$ & $103.9-111.6$ & $197.9-214.8$ & $159.2-185.3$ & $324.2-452.2$ \\
& $(41.9-55.7)$ & $(72.5-121.3)$ & $(70.7-120.7)$ & $(305.2-415.1)$ \\
$\max _{2 \mid}$ & $12.7(23.1)$ & $8.2(12.3)$ & $23.7(84.3)$ & $29.9(75.9)$ \\
\hline \hline
\end{tabular}




\section{FIGURE CAPTIONS}

Fig. 1. The regions in the unification scale parameters $\left(m_{o}, M_{o}\right)$, obtained by demanding radiative gauge symmetry breaking, in form of the scatter plots obtained by the scanning procedure described in text. These are 2-dimensional projections of the solutions in the 5-dimensional parameter space. Crosses: solutions already excluded by experimental constraints discussed in the text; squares: solutions compatible with experimental constraints with $\max \left|\Delta_{i j}\right|<$ 30; dots: solutions compatible with experimental constaints with $30<\max \left|\Delta_{i j}\right|$ $<100$. Dashed contours: solutions with $\max \left|\Delta_{i j}\right|<10$. Solid contours: solutions compatible with experimental constraints and with $\max \left|\Delta_{i j}\right|<30$, but with no radiative corrections.
A) $M_{t}=130 \mathrm{GeV}, \tan \beta=2$; B) $M_{t}=130 \mathrm{GeV}, \tan \beta=10$;
C) $M_{t}=180 \mathrm{GeV}, \tan \beta=2$; D) $M_{t}=180 \mathrm{GeV}, \tan \beta=30$.
In case $\mathrm{C}$ ) there are no solutions corresponding to the contour criteria.

Fig. 2. Same as Fig. 1 for $\left(m_{o}, A_{o}\right)$.

Fig. 3. Same as Fig. 1 for $\left(A_{o} M_{o}\right)$.

Fig. 4. Same as Fig. 1 for $\left(B_{o} / m_{o}, A_{o} / m_{o}\right)$.

Fig. 5. Same as Fig. 1 for the low energy parameters $\left(M_{2}, \mu\right)$.

Fig. 6. Same as Fig. 1 for the physical lightest neutralino mass $M_{n}$ and the pseudoscalar mass $M_{A}$.

Fig. 7. Same as Fig. 1 for the gaugino content of the lightest neutralino, $Z_{n}=Z^{2}(1,1)+Z^{2}(1,2)$ and its mass $M_{n}$.

Fig. 8. Same as Fig. 1 for the lighter chargino mass $M_{c}$ and the pseudoscalar mass $M_{A}$.

Fig. 9. Same as Fig. 1 for the lighter chargino mass $M_{c}$ and the lightest neutralino mass $M_{n}$.

Fig. 10. Same as Fig. 1 for the physical lightest stop mass $m_{\tilde{t}}$ and the universal gaugino mass at $M_{X}, M_{o}$.

Fig. 11. Same as Fig. 1 for the physical stau mass $m_{\tilde{\tau}}$ and the universal scalar mass at $M_{X}, m_{o}$.

Fig. 12. Same as Fig. 1 for the stop mass $m_{\tilde{t}}$ and the pseudoscalar mass $M_{A}$.

Fig. 13. Same as Fig. 1 for the chargino mass $M_{c}$ and the stop mass $m_{\tilde{t}}$.

Fig. 14. Solutions for $M_{t}=130 \mathrm{GeV}$ and $\tan \beta=30$. Notation as in Fig. 1 (dots correspond now to solutions with $\left.30<\max \left|\Delta_{i j}\right|<250\right)$. The corresponding value of $Y_{t} / Y_{b}$ at $M_{X}$ is 1.08 . 
Fig. 15. Solutions for $M_{t}=130 \mathrm{GeV}$ and $\tan \beta=31$. There only exist solutions with $\max \left|\Delta_{i j}\right|>30$. Plotted are solutions with $\max \left|\Delta_{i j}\right|<500$. The corresponding values of $Y_{t} / Y_{b}$ at $M_{X}$ is 1.04 .

Fig. 16. Same as Fig. 15 for $M_{t}=180 \mathrm{GeV}$ and $\tan \beta=50$. The corresponding values of $Y_{t} / Y_{b}$ at $M_{X}$ is 1.18 .

Fig. 17. Same as Fig. 15 for $M_{t}=180 \mathrm{GeV}$ and $\tan \beta=51$. The corresponding values of $Y_{t} / Y_{b}$ at $M_{X}$ is 1.12. Plotted are solutions with $\max \left|\Delta_{i j}\right|<750$. 